Pacific Journal of Mathematics

THE TWO-DIMENSIONAL ANALOGUE OF THE CATENARY

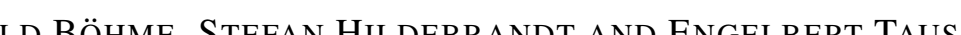




\title{
THE TWO-DIMENSIONAL ANALOGUE OF THE CATENARY
}

\author{
Reinhold Böhme, Stefan Hildebrandt, \\ AND ENGELBERT TAUSCH
}

In this paper we consider the following isoperimetric problem: let $\Gamma$ be a prescribed Jordan curve in $R^{3}$. Determine a surface of given area spanning $\Gamma$, which has minimal potential energy under gravitational forces. This will be of importance for the construction of "perfect domes."

1. Introduction. Ever since the derivation of the law of refraction light by Pierre Fermat in 1662, the one-dimensional problem

$$
\int \omega(x, y, z) \sqrt{d x^{2}+d y^{2}+d z^{2}} \longrightarrow \text { Extreme }
$$

has been of great interest. According to Fermat's principle, this variational problem governs the propagation of light in an isotropic but inhomogeneous medium where $v=1 / \omega$ is the velocity of the light particle. On the other hand, the motion of a mass particle in a conservative force field grad $U$ can also be described by an extremal problem (1.1) where $\omega=\sqrt{2(U+h)}$, according to Maupertuis' principle of least action as it has been formulated by Jacobi.

As is well known, the modern history of calculus of variations started with the brachystochrone, the problem of quickest descent, proposed by John Bernoulli in 1696 [2]. It consists in minimizing the integral

$$
\int \frac{1}{\sqrt{z}} \sqrt{d x^{2}+d z^{2}}
$$

which is a special case of the integral

$$
\int \omega(x, z) \sqrt{d x^{2}+d z^{2}}
$$

treated by Leonhard Euler in his classical treatise "methodus inveniendi lineas curvas ..." in 1744. In particular, he dealt with the cases $\omega(x, z)=1,1 / \sqrt{x}, x z, x^{n},\left(x^{2}+z^{2}\right)^{n}, z^{n} / z^{m}, \lambda+z$.

An excellent discussion of these and of other important examples can be found in the well known lectures by Bolza [5].

The integral

$$
\int z \sqrt{d x^{2}+d z^{2}}, \quad z>0,
$$

leads to the celebrated problem, to determine the surfaces of revolu- 
tion minimizing area. The regular extremals of (1.4) are the catenaries

$$
z=a \cosh \left(\frac{x-x_{0}}{a}\right) .
$$

They are closely related to the following isoperimetric problem: What are the curves of given length connecting two points $P_{1}$ and $P_{2}$ in the $x, z$-plane which have the lowest center of gravity? Introducing a Lagrange multiplier $\mu$, one is led to the problem

$$
\int(z+\mu) \sqrt{d x^{2}+d z^{2}} \longrightarrow \min
$$

which, clearly, can be reduced to the minimization of (1.4). Thus the catenary describes also the equilibrium position of a heavy chain.

The two-dimensional analogue has only recently found some interest although the nonparametric version

$$
\int \omega(x, y, z) \sqrt{1+z_{x}^{2}+z_{y}^{2}} d x d y \longrightarrow \min
$$

had already been considered by Jellett [16] in 1850. To our knowledge, the first existence results for (1.6) with nonconstant $\omega$ are due to Tausch [20]. Let us now consider the parametric problem

$$
\int \omega\left(\mathfrak{x}_{0}\right)\left|\mathfrak{x}_{. u} \times \mathfrak{x}_{v}\right| d u d v \longrightarrow \min
$$

for $\mathfrak{x}=\mathfrak{x}(u, v)=(x(u, v), y(u, v), z(u, v))$. Introducing conformal parameters, $u, v$, we have

$$
\left|\mathfrak{x}_{u} \times \mathfrak{x}_{v}\right|=\frac{1}{2}\left\{\mathfrak{x}_{u}^{2}+\mathfrak{x}_{v}^{2}\right\}
$$

while in general,

$$
\left|\mathfrak{x}_{u} \times \mathfrak{x}_{v}\right| \leqq \frac{1}{2}\left\{\mathfrak{x}_{u}^{2}+\mathfrak{x}_{v}^{2}\right\}
$$

Hence, we may replace (1.7) by the Plateau problem for the generalized Dirichlet integral

$$
J(\mathfrak{x})=\int \omega(\mathfrak{x})|\nabla \mathfrak{r}|^{2} d u d v
$$

belonging to the special metric

$$
d s^{2}=\omega(\mathfrak{x})|d \mathfrak{x}|^{2}=\omega(\mathfrak{x})\left\{d x^{2}+d y^{2}+d z^{2}\right\} .
$$

It is a special case of the Dirichlet integral 


$$
\int g_{i j}\left(\mathfrak{r}_{)}\right)\left\{x_{u}^{i} x_{u}^{j}+x_{v}^{i} x_{v}^{j}\right\} d u d v \text {. }
$$

The Plateau problem for this functional has been solved by Morrey [18] (cf. also [19] and [15]) for a fairly large class of positive definite metrics

$$
d s^{2}=g_{i j}(\mathfrak{x}) d x^{i} d x^{j}
$$

The conformality relations associated with (1.10) are

$$
g_{i j}(\mathfrak{x}) x_{u}^{i} x_{u}^{j}=g_{i j}\left(\mathfrak{x}_{)}\right) x_{v}^{i} x_{v}^{j} ; \quad g_{i j}\left(x_{i}\right) x_{u}^{i} x_{v}^{j}=0 .
$$

However, for important examples, the metric $d s^{2}$ is only semidefinite, or even indefinite. For instance, the two-dimensional analogue of the catenary problem (1.4) leads to

$$
\int z|\nabla \mathfrak{x}|^{2} d u d v \longrightarrow \min
$$

To get a regular problem we have to restrict the surfaces to the upper half space $\{z>0\}$. That is, we have to minimize (1.8) or (1.10) under Plateau boundary conditions as well as under obstacle conditions. A general approach to obstacle problems for (1.10) has been given in [21], [15], [13], and [14]. In the present paper, we shall treat obstacle problems for (1.8), and, in more detail, the obstacle problem $z>0$ for (1.13).

Professor Frei Otto suggested to us the following isoperimetric problem: Let $\Gamma$ be a prescribed Jordan curve in $\boldsymbol{R}^{3}$. Determine a surface of given area spanning $\Gamma$, which has minimal potential energy under gravitational forces, that is, a surface having the lowest center of gravity.

Hence, we look for a mapping $x: \overline{\mathscr{B}} \rightarrow R^{3}$ of the closure of the unit disc in a $u, v$-plane into $\boldsymbol{R}^{3}$, which maps $\partial \mathscr{P}$ monotonicly onto $\Gamma$, such that

$$
\int_{s} z\left|\mathfrak{x}_{u} \times \mathfrak{x}_{v}\right| d u d v
$$

assumes a minimum while the value of

$$
\int\left|x_{u} \times x_{v}\right| d u d v
$$

is a prescribed number.

Introducing a Lagrange multiplier $\mu$, we can replace this problem by the following simpler one:

Minimize 


$$
\int_{\mathscr{B}}(z+\mu)\left|\mathfrak{x}_{u} \times x_{v}\right| d u d v
$$

among all surfaces $r: \overline{\mathscr{B}} \rightarrow \boldsymbol{R}^{3}$ spanning $\Gamma$, and such, that $z+\mu \geqq 0$.

Using the argument mentioned in connection with (1.7) we can replace (1.14) by

$$
\int_{\mathscr{O}}(z+\mu)|\nabla \mathfrak{r}|^{2} d u d v
$$

which, by an obvious coordinate transformation, leads us to the problem (1.13).

In the following, we shall concentrate on the discussion of this variational problem which, according to a remark by Professor Frei Otto, will be of importance for the construction of "perfect domes." We wish to thank him for proposing this question to us. Furthermore, we are very grateful to Prof. Ernst Hölder, who drew our attention to the variational problem (1.7) and to the formula (2.13), which had been derived for the nonparametric case by Jellet [16]. The analogue for the one-dimensional problem (1.3) is due to Gauß (cf. [4], pp. 84-85).

Outline of the paper. In $\S 2$, we discuss the Euler equations for the variational integral

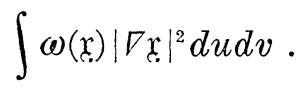

In $\S 3$, we list the existence and regularity results for the solutions of the Plateau problem for (1.16) with an obstacle as additional side condition. A variant of the maximum principle due to Chicco is presented.

In the following three sections, various inclusion theorems for the solutions of obstacle problems for

$$
\int z|\nabla r|^{2} d u d v
$$

are derived. These inclusion theorems yield conditions guaranteeing that the solution surfaces do not touch the boundary of the obstacle, and thus that they satisfy the Euler equations of (1.17) at all points. Therefore, the solutions are the perfect two-dimensional analogues of the catenaries. Combining the variational approach with a constructive method, say, with the finite element method, it should be possible to compute the solutions. The inclusion theorems yield a general impression of the shape of the solution surfaces. Most of the inclusion results are derived from Chicco's maximum principle, 
except for the results of $\S 6$ which are based on a technique due to Allard [1], and Michael and Simon [17].

We conclude our discussion with a remark on "perfect domes."

For useful suggestions and remarks, we wish to thank professors E. Giusti, R. Finn, E. Hölder, K. Steffen, and H. C. Wente.

2. The Euler equations for $\int \omega(\mathfrak{x})|\nabla \mathfrak{x}|^{2} d u d v$. The Euler equations for the integral (1.8) are

$$
\partial_{u}\left\{2 \omega(\mathfrak{x}) \partial_{u} \mathfrak{x}\right\}+\partial_{v}\left\{2 \omega(\mathfrak{x}) \partial_{v} \mathfrak{x}\right\}=\omega^{\prime}(\mathfrak{x})|\nabla \mathfrak{x}|^{2}
$$

where

$$
\partial_{u}=\frac{\partial}{\partial u}, \quad \partial_{v}=\frac{\partial}{\partial v}, \quad \omega^{\prime}=\omega \mathrm{r}=\operatorname{grad}_{\imath} \omega .
$$

For the sake of simplicity, we write $\omega$ instead of $\omega(x)$ or $\omega \circ x$, similarly $\omega^{\prime}$ instead of $\omega^{\prime}($ r. $)$ or $\omega^{\prime} \circ$ r., etc., whenever no misunderstanding can occur. Equation (2.1) is equivalent to

$$
2 \omega \Delta \mathfrak{x}=\omega^{\prime}|\nabla \mathfrak{x}|^{2}-2 \omega_{x \jmath}\left\{x_{u}^{j} \mathfrak{x}_{u}+x_{v}^{j} \mathfrak{x}_{v}\right\}, \quad j=1,2,3,
$$

or

$$
\Delta \mathfrak{x}=\frac{1}{2} \frac{\omega^{\prime}}{\omega}|\nabla \mathfrak{r}|^{2}-\left\{\frac{\omega^{\prime}}{\omega} \cdot \mathfrak{x}_{u}\right\} \mathfrak{r}_{u}-\left\{\frac{\omega^{\prime}}{\omega} \cdot \mathfrak{r}_{v}\right\} \mathfrak{x}_{v} .
$$

Introducing the vector valued function

$$
k=\frac{\omega^{\prime}}{\omega}=\operatorname{grad}_{\varepsilon} \log \omega
$$

we can rewrite $(2.3)$ as

$$
\Delta \mathfrak{r}=\frac{1}{2} k|\nabla \mathfrak{c}|^{2}-\left(k \cdot \mathfrak{r}_{u}\right)_{\mathrm{u} u}-\left(k \cdot \mathfrak{\Upsilon}_{v}\right) \mathfrak{\varkappa}_{v} .
$$

The conformality relations $(1.12)$ reduce to

$$
\mathfrak{c}_{u}^{2}=\mathfrak{x}_{v}^{2}, \quad \mathfrak{x}_{u} \cdot \mathfrak{c}_{v}=0 .
$$

In virtue of (2.6), and of the identity

$$
\mathfrak{z} \times(\mathfrak{a} \times \mathfrak{b})=(\mathfrak{z} \cdot \mathfrak{b}) \mathfrak{a}-(\mathfrak{z} \cdot \mathfrak{a}) \mathfrak{b}
$$

which holds for arbitrary vectors $z, a, \mathfrak{b} \in \boldsymbol{R}^{3}$, we obtain

$$
\left\{k \cdot \frac{\mathfrak{x}_{u} \times \mathfrak{x}_{v}}{\left|\mathfrak{x}_{u} \times \mathfrak{x}_{v}\right|}\right\}\left[\mathfrak{x}_{u} \times \mathfrak{x}_{v}\right]=\frac{\mathfrak{x}_{u} \times \mathfrak{x}_{v}}{\left|\mathfrak{x}_{u} \times \mathfrak{r}_{v}\right|} \times\left(\left[\mathfrak{x}_{u} \times \mathfrak{x}_{v}\right] \times k\right)+\frac{1}{2}\left|\nabla \mathfrak{x}_{v}\right|^{2} k,
$$


and

$$
\left[\mathfrak{x}_{u} \times \mathfrak{x}_{v}\right] \times k=\left(k \cdot \mathfrak{x}_{u}\right) \mathfrak{x}_{v}-\left(k \cdot \mathfrak{x}_{v}\right) \mathfrak{x}_{u},
$$

whence

$$
\begin{aligned}
{\left[\mathfrak{x}_{u} \times \mathfrak{x}_{v}\right] \times\left(\left[\mathfrak{x}_{u} \times \mathfrak{x}_{v}\right] \times k\right)=} & \left(k \cdot \mathfrak{x}_{u}\right)\left[\mathfrak{x}_{u} \times \mathfrak{x}_{v}\right] \times \mathfrak{x}_{v} \\
& -\left(k \cdot \mathfrak{x}_{v}\right)\left[\mathfrak{x}_{u} \times \mathfrak{x}_{v}\right] \times \mathfrak{x}_{u} \\
= & -\left\{\left(k \cdot \mathfrak{x}_{u}\right) \mathfrak{x}_{u}+\left(k \cdot \mathfrak{x}_{v}\right) \mathfrak{x}_{v}\right\} \frac{1}{2}\left|\nabla \mathfrak{r}^{2}\right|^{2}
\end{aligned}
$$

and therefore,

$$
\frac{\mathfrak{r}_{u} \times \mathfrak{x}_{v}}{\left|\mathfrak{x}_{u} \times \mathfrak{x}_{v}\right|} \times\left(\left[\mathfrak{x}_{u} \times \mathfrak{x}_{v}\right] \times k\right)=-\left(k \cdot \mathfrak{x}_{u}\right) \mathfrak{x}_{u}-\left(k \cdot \mathfrak{x}_{v}\right) \mathfrak{x}_{v} .
$$

By (2.8) and (2.9),

$$
\left\{k \cdot \frac{\mathfrak{x}_{u} \times \mathfrak{x}_{v}}{\left|\mathfrak{x}_{u} \times \mathfrak{x}_{v}\right|}\right\}\left[\mathfrak{x}_{u} \times \mathfrak{x}_{v}\right]=\frac{1}{2}|\nabla \mathfrak{x}|^{2} k-\left(k \cdot \mathfrak{x}_{u}\right) \mathfrak{x}_{u}-\left(k \cdot \mathfrak{r}_{v}\right) \mathfrak{x}_{v} .
$$

Defining the surface normal $\mathfrak{N}$ by

$$
\mathfrak{l}=\frac{\mathfrak{r}_{u} \times \mathfrak{r}_{v}}{\left|\mathfrak{r}_{u} \times \mathfrak{x}_{v}\right|},
$$

the Euler equation (2.1) takes the form

$$
\Delta \mathfrak{r}=\left(k \cdot \mathfrak{N}_{i}\right)\left[\mathfrak{x}_{u} \times \mathfrak{x}_{v}\right] .
$$

On account of (2.6), equation (2.12) shows that the extremals $\mathfrak{x}=\mathfrak{x}(u, v)$ of $(1.8)$ are surfaces with mean curvature

$$
\mathscr{K}(\mathfrak{x})=\frac{1}{2} k(\mathfrak{x}) \cdot \mathfrak{N}=\frac{1}{2} \frac{\partial}{\partial \mathfrak{R}} \log \omega .
$$

Hence, we have the following interpretations of (2.1):

(i) The extremals of (1.8) are minimal surfaces with respect to the metric (1.9).

(ii) The extremals of (1.8) are surfaces having the mean curvature (2.13) depending on the position vector $x$ as well as on the surface normal $\mathfrak{x}$.

According to these two interpretations, different maximum principles can be derived.

3. Existence and regularity of solutions of the Plateau problem for $\int_{\mathscr{S}} \omega(\mathfrak{x})|\nabla \mathfrak{r}|^{2} d u d v$. Let $\mathscr{C}$ be a closed set in $\boldsymbol{R}^{3}$, which is the closure of its interior, and suppose that $\omega=\omega(\mathfrak{r})$ is a function of class $C^{2}(\mathscr{Y}, \boldsymbol{R})$ with $\omega(\mathfrak{x}) \geqq \varepsilon>0$ for all $x \in \%$. By $\Gamma$ we denote 
a closed rectifiable Jordan curve contained in int $\mathscr{K}$. Define the class $\mathfrak{S}(\Gamma)$ by

$$
\begin{aligned}
\mathfrak{E}(\Gamma):= & \left\{\mathfrak{c} \in H_{2}^{1}\left(\mathscr{B}, R^{3}\right): \mathfrak{x}(u, v) \in \mathscr{K} \text { for almost all }(u, v) \in \mathscr{B}\right. \text {, and } \\
& \mathfrak{x}: \partial \mathscr{B} \rightarrow \Gamma \text { is a weakly monotonic, continuous map of } \\
& \partial \mathscr{B} \text { onto } \Gamma\} .
\end{aligned}
$$

For $\mathfrak{x} \in \mathbb{S}(\Gamma)$, we can define the integral

$$
J(\mathfrak{c})=\int_{\mathscr{D}} \omega(\mathfrak{x})|\nabla \mathfrak{x}|^{2} d u d v
$$

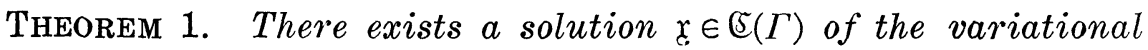
problem

$$
\mathfrak{B}(\Gamma): J \longrightarrow \min \text { on } \mathfrak{S}(\Gamma) \text {. }
$$

Every solution of $\mathfrak{P}(\Gamma)$ satisfies a.e. on $\mathscr{B}$ the conformality relations

$$
\mathfrak{x}_{u}^{2}=\mathfrak{x}_{v}^{2}, \mathfrak{x}_{u} \cdot \mathfrak{x}_{v}=0 \text {. }
$$

In addition, if $\mathscr{K}$ is quasiregular (cf. [15]), each solution of $\mathfrak{P}(\Gamma)$ is Hölder continuous on $\mathscr{B}$, and continuous on $\overline{\mathscr{B}}$.

Proof. The result can be proved by a technique due to Morrey. A complete proof is presented in [15], in particular, pp. 198-200.

THeOREM 2. If $\mathscr{K}$ is a quasiregular set of class $C^{3}$, each solution of $\mathfrak{P}(\Gamma)$ is of class $H_{s, \mathrm{loc}}^{2} \cap C^{1, \alpha}\left(\mathscr{R}, \boldsymbol{R}^{3}\right)$, for all $s \in[1, \infty)$, and for all $\alpha \in(0,1)$.

Proof. Cf. [13, 14, 20].

THEOREM 3. If $\Gamma$ is a regular curve of class $C^{2, \beta}, 0<\beta<1$, and $\mathscr{K}$ is quasiregular and of class $C^{3}$, each solution of $\mathfrak{B}(\Gamma)$ is of class $H_{s}^{2}\left(\mathscr{B}, \boldsymbol{R}^{3}\right) \cap C^{1, \alpha}\left(\overline{\mathscr{B}}, \boldsymbol{R}^{3}\right), s \in[1, \infty)$ and $\alpha \in(0,1)$, and it is of class $C^{2, \beta}$ in a sufficiently small strip around $\partial \mathscr{B}$.

Proof. Because of Theorem 2, it suffices to prove the last statement. Since $\Gamma$ is assumed to lie in int $\mathscr{K}$, and since $\mathfrak{r} \in C^{\circ}\left(\overline{\mathscr{B}}, \boldsymbol{R}^{3}\right)$ in virtue of Theorem $1, \mathfrak{x}$ satisfies the Euler equation for $J$ in a sufficiently small strip around $\partial \mathscr{B}$. Then, [11] can be applied.

In the following, we suppose that the assumptions of Theorem 3 are satisfied. For a solution $\mathfrak{r}$ of $\mathfrak{P}(\Gamma)$, we introduce the "touching set" 


$$
\mathscr{T}=\{(u, v) \in \mathscr{B}: \mathfrak{d}(u, v) \in \partial \mathscr{K}\},
$$

which is mapped by $x$ onto the boundary of $\mathscr{L}$; and its open complement

$$
\mathscr{B} \mathscr{B}_{0}=\mathscr{B}-\mathscr{T}
$$

which is mapped into int $\mathscr{K}$.

On $\mathscr{B}_{0}, \mathfrak{r}(u, v)$ satisfies the Euler equation

$$
\Delta \mathfrak{x}=\frac{1}{2} k|\nabla \mathfrak{x}|^{2}-\left(k \cdot \mathfrak{r}_{u}\right) \mathfrak{x}_{u}-\left(k \cdot \mathfrak{x}_{v}\right) \mathfrak{x}_{v}
$$

where

$$
k=\frac{1}{\omega} \quad \omega^{\prime}=\frac{1}{\omega} \operatorname{grad}_{\varepsilon} \omega=\operatorname{grad}_{\varepsilon} \log \omega
$$

or, equivalently,

$$
\Delta \mathfrak{r}=(k \cdot \mathfrak{R})\left[\mathfrak{x}_{u} \times \mathfrak{x}_{v}\left[\quad \text { on } \mathscr{B} \mathscr{B}_{0} .\right.\right.
$$

But on $\mathscr{F}, \mathfrak{c}(u, v)$ satisfies a.e. the equation

$$
\Delta \mathfrak{x}=2 \mathscr{H}^{*}(\mathfrak{x})\left[\mathfrak{x}_{u} \times \mathfrak{x}_{v}\right]
$$

when $\mathscr{H}^{*}(\mathfrak{x})$ denotes the mean curvature of $\partial \mathscr{K}$ in the point $x$. In particular, we have

$$
\Delta \mathfrak{x}=0 \quad \text { a.e. on } \mathscr{T}^{-}
$$

where $\partial \mathscr{K}$ is a plane.

To each solution $x$ of $\$(\Gamma)$, we form the linear operator

$$
L=\Delta+\Lambda
$$

where

$$
\Lambda=a(u, v) \partial_{u}+b(u, v) \partial_{v}
$$

and where

$$
a(u, v)=k(\mathfrak{x}(u, v)) \mathfrak{x}_{u}(u, v), \quad b(u, v)=k(\mathfrak{\complement}(u, v)) \mathfrak{x}_{v}(u, v) .
$$

That is,

$$
L=\Delta+\left\{k(\mathfrak{x}) \cdot \mathfrak{x}_{u}\right\} \partial_{u}+\left\{k(\mathfrak{c}) \cdot \mathfrak{c}_{v}\right\} \partial_{v} .
$$

Because of Theorem 3, the functions $a$ and $b$ are certainly of class $L^{\infty}(\mathscr{B})$. The Euler equation (3.1) can now also be written as

$$
L \mathrm{r}=\frac{1}{2} k(\mathfrak{x})|\nabla \mathfrak{x}|^{2} .
$$


By an observation due to Chicco, $H_{2}^{1}(\mathscr{B}, \boldsymbol{R})$-subsolutions of $L$ satisfy the strong maximum principle, i.e., if $\sigma \in H_{2}^{1}(\mathscr{B}, \boldsymbol{R})$ fulfills

$$
\int\{\nabla \sigma \cdot \nabla \varphi+\Lambda \sigma \cdot \varphi\} d u d v \leqq 0 \text { for all } \varphi \in \stackrel{\circ}{H}_{2}^{1}(\mathscr{B}, \boldsymbol{R}) \text { with } \varphi \geqq 0 \text {, }
$$

we have

$$
\sup _{\mathscr{S}} \sigma \leqq \sup _{\partial \mathscr{B}} \sigma \text {, }
$$

and $\sup _{\mathscr{\mathscr { O }}} \sigma=\sup _{\mathscr{B}} \sigma$ for some ball $\mathscr{B}^{\prime} \subset \subset \mathscr{B}$ implies that $\sigma=$ const a.e. on $\mathscr{B}$. (Here, sup means the essential supremum; cf. [6], and also [8], Theorems 8.1 and 8.19, pp. 168 and 188-189.)

This maximum principle can also be formulated in the following way:

Lemma 1. If we have $\sigma \in C^{0}(\overline{\mathscr{B}}, \boldsymbol{R})$, and

$$
L \sigma \geqq 0 \quad \text { a.e. on } \mathscr{B} \text {, }
$$

then

$$
\max _{s} \sigma=\max _{\partial \phi} \sigma
$$

and $\sigma$ assumes its maximum on $\overline{\mathscr{B}}$ in an interior point of $\mathscr{B}$ if and only if $\sigma \equiv$ const.

REMARK. Note that each composition $\sigma=f \circ \mathfrak{\gamma}$ of the considered solution of $\mathfrak{B}(\Gamma)$ with a $C^{2}(\mathscr{K}, \boldsymbol{R})$-function $f$ is of class $C^{0}(\overline{\mathscr{B}}, \boldsymbol{R}) \cap$ $H_{2}^{2}(\mathscr{B}, \boldsymbol{R})$ by virtue of Theorem 3 , so that we can apply the lemma to such a composition.

4. Inclusion theorems for the solutions of $\mathfrak{B}(\Gamma)$ when $J(\mathfrak{\complement})=$ $\int z|\nabla \mathfrak{c}|^{2} d u d v$, and when $\mathscr{K}$ reduces to $\{\mathfrak{x}: z>\varepsilon\}, \varepsilon>0$. Let $\omega(\mathfrak{r})=z$, thus, $\omega^{\prime}=(0,0,1)$, and

$$
k(x)=\left(0,0, \frac{1}{z}\right) .
$$

Suppose that

$$
\mathscr{K}:=\{\mathfrak{s}: z \geqq \varepsilon\}, \quad \varepsilon>0,
$$

and that $\Gamma \subset\{\mathfrak{c}: z>\varepsilon\}$.

Let $\mathfrak{x}=\mathfrak{r}(u, v)$ be a solution of $\mathfrak{A}(\Gamma)$. The associated operator $L=\Delta+\Lambda$ is now given by 


$$
a=\frac{z_{u}}{z}, \quad b=\frac{z_{v}}{z}, \quad \Lambda=a \hat{\partial}_{u}+b \partial_{v} .
$$

(Note that $z_{u}=z_{v}=z_{u u}=z_{u v}=z_{v v}=0$ a.e. on . .)

Furthermore, by (3.3) and (3.4), we obtain that the Euler (in)equalities take the form

$$
\begin{aligned}
\Delta x & =-\frac{1}{z}\left\{x_{u} z_{u}+x_{v} z_{v}\right\} \\
\Delta y & =-\frac{1}{z}\left\{y_{u} z_{u}+y_{v} z_{v}\right\} \\
\Delta z & =-\frac{1}{z}\left\{z_{u}^{2}+z_{v}^{2}\right\}+\frac{1}{2 z}|\nabla \mathfrak{x}|^{2} \text { on } \mathscr{B}_{0},=0 \text { a.e. on } \mathscr{T} .
\end{aligned}
$$

Equivalently, we have

$$
\begin{aligned}
\Delta x & =-\Lambda x \\
\Delta y & =-\Lambda y \\
\Delta z & =-\Lambda z+\frac{1}{2 z}|\nabla x|^{2} \text { on } \mathscr{B}_{0},=0 \text { a.e. on } \mathscr{S} .
\end{aligned}
$$

By (3.2), this can be written as

$$
\begin{aligned}
& \Delta x=\frac{1}{z W} \cdot \xi \cdot \zeta, \\
& \Delta y=\frac{1}{z W} \cdot \eta \cdot \zeta, \\
& \Delta z=\frac{1}{z W} \cdot \zeta^{2} \text { on } \mathscr{B}_{0},=0 \text { on } \mathscr{T},
\end{aligned}
$$

if $W \neq 0$, where

$$
\begin{gathered}
\mathfrak{x}_{u} \times \mathfrak{r}_{v}=(\xi, \eta, \zeta), \quad \text { and } \\
W=\left|\mathfrak{r}_{u} \times \mathfrak{x}_{v}\right|=\sqrt{\xi^{2}+\eta^{2}+\zeta^{2}}=\frac{1}{2}|\nabla \mathfrak{r}|^{2}=\mathfrak{x}_{u}^{2}=\mathfrak{x}_{v}^{2} .
\end{gathered}
$$

Clearly, the third equation of (4.6) implies that $z$ is subharmonic on $\mathscr{B}$ whence

$$
z(u, v) \leqq \max _{\partial \mathscr{B}} z \text { for all }(u, v) \in \mathscr{B} .
$$

This follows also from the third equation of (4.4) by means of the conformality relations. In fact,

$$
\mathfrak{x}_{u}^{2}=\mathfrak{x}_{v}^{2}, \quad \mathfrak{x}_{u} \cdot \mathfrak{x}_{v}=0
$$

implies that 


$$
\mathfrak{x}_{w}^{2}=0, \text { where we have set } \mathfrak{x}_{w}=\frac{1}{2}\left(\mathfrak{x}_{u}-i \mathfrak{x}_{v}\right)
$$

whence

$$
|\nabla \boldsymbol{z}|^{2} \leqq|\nabla x|^{2}+|\nabla y|^{2}
$$

Therefore,

$$
|\nabla z|^{2} \leqq \frac{1}{2}|\nabla x|^{2}
$$

whence, by (4.4),

$$
\Delta z \geqq 0 \quad \text { on } \mathscr{B} .
$$

Furthermore, let us introduce

$$
\mathfrak{r}(u, v)=(x(u, v), y(u, v), 0)
$$

which is the orthogonal projection of $\mathfrak{x}(u, v)$ onto the plane $\{z=0\}$. Then, (4.9) becomes

$$
|\nabla \boldsymbol{z}|^{2} \leqq|\nabla \mathfrak{x}|^{2} \text { in } \mathscr{B},
$$

and, secondly, we infer from (4.5) that

$$
L \mathfrak{x}=0 \text { in } \mathscr{B} .
$$

Hence, $\mathfrak{r}(\overline{\mathscr{B}})$ is contained in the convex hull $\hat{\mathfrak{Z}}_{\Gamma}$ of the cylinder $3_{\Gamma}:=\gamma \times\{z$-axis $\}, \gamma$ being the orthogonal projection of $\Gamma$ onto the plane $\{z=0\}$.

Combining this result with (4.8) we arrive at the following first inclusion theorem:

THEOREM 4. Let $\mathfrak{x}(u, v)$ be a solution of the variational problem $\mathfrak{B}(\Gamma)$. Then, for each $(u, v) \in \mathscr{B}$, the point $\mathrm{x}(u, v)$ is contained in the cylinder int $\hat{\mathfrak{Z}}_{\Gamma} \cap\left\{(x, y, z): \varepsilon \leqq z \leqq h_{\Gamma}\right\}$ where

$$
h_{\Gamma}=\max \{z(u, v):(u, v) \in \partial \mathscr{B}\}
$$

denotes the maximal z-component of $\Gamma$.

Next, we note the identities

$$
\Lambda \varphi^{2}=2 \varphi \Lambda \varphi
$$

and

$$
\Delta \varphi^{2}=2|\nabla \phi|^{2}+2 \varphi \Delta \phi
$$

holding for arbitrary functions $\varphi \in C^{2}(\mathscr{B}, \boldsymbol{R})$. Let $\mathfrak{x}_{0}=\left(x_{0}, y_{0}, z_{0}\right)$ be 
an arbitrary constant point, and let $\mathfrak{x}_{0}=\left(x_{0}, y_{0}, 0\right)$. On account of (4.5), a straightforward calculation shows that

$$
L\left(\mathfrak{x}-\mathfrak{x}_{0}\right)^{2}=2|\nabla \mathfrak{x}|^{2}
$$

and

$$
L\left(z-z_{0}\right)^{2}=\left\{\begin{array}{l}
2|\nabla z|^{2}+\frac{z-z_{0}}{z}|\nabla \mathfrak{x}|^{2} \text { on } \mathscr{B}, \\
2|\nabla z|^{2}
\end{array}\right.
$$

Hence, for some real parameter $c>0$,

$$
\begin{aligned}
L\left\{\left(\mathfrak{x}-\mathfrak{r}_{0}\right)^{2}+\frac{1}{c^{2}}\left(z-z_{0}\right)^{2}\right\} & \\
& =2|\nabla \mathfrak{x}|^{2}+\frac{2}{c^{2}}|\nabla z|^{2}+\left\{\begin{array}{cr}
\frac{1}{c^{2}} \frac{z-z_{0}}{z}|\nabla \mathfrak{x}|^{2} & \text { on } \mathscr{B}_{0}, \\
0 & \text { a.e. on } \mathscr{T} .
\end{array}\right.
\end{aligned}
$$

Suppose that $z_{0}>0,0<\varepsilon \leqq z$, and that $2 c^{2}+1-z_{0} / z \geqq 0$. Using (4.12), the right hand side of the inequality (4.19) can be estimated from below by

$$
\frac{2}{c^{2}}\left[c^{2}+2-\frac{z_{0}}{z}\right]|\nabla z|^{2} \text { or } 0, \text { on } \mathscr{B} \text { or on } \mathscr{T} \text {, respectively. }
$$

Thus we infer

LeMmA 2. If $0<\varepsilon \leqq z<z_{0}$, and if $z_{0} / \varepsilon \leqq \min \left\{2 c^{2}+1, c^{2}+2\right\}$, then

$$
L\left\{\left(\mathfrak{x}-\mathfrak{r}_{0}\right)^{2}+\frac{1}{c^{2}}\left(z-z_{0}\right)^{2}\right\} \geqq 0 \text { a.e. on } \mathscr{B} \text {. }
$$

Next, we consider the composition

$$
\sigma=\left(\mathfrak{x}-\mathfrak{x}_{0}\right)^{2}-\frac{1}{c^{2}}\left(z-z_{0}\right)^{2}
$$

for $c \geqq 1$ and $z_{0} \geqq 0$. Using (4.12), (4.17), and (4.18), we obtain that

$$
L \sigma \geqq \frac{2}{c^{2}}\left(c^{2}-1\right)|\nabla \mathfrak{x}|^{2} \geqq 0 \text { on } \mathscr{T},
$$

and that

$$
L \sigma \geqq \frac{2}{c^{2}}\left[c^{2}-2+\frac{z_{0}}{z}\right]|\nabla z|^{2} \text { a.e. on } \mathscr{B}_{0}
$$


Hence, we arrive at

Lemma 3. If $z_{0} \geqq 0,0<\varepsilon \leqq z \leqq h_{\Gamma}$, and if

$$
c \geqq 1 \quad \text { and } \quad c^{2}-2+\frac{z_{0}}{h_{\Gamma}} \geqq 0 ，
$$

then we have

$$
L\left\{\left(\mathfrak{x}-\mathfrak{x}_{0}\right)^{2}-\frac{1}{c^{2}}\left(z-z_{0}\right)^{2}\right\} \geqq 0
$$

Thirdly, we consider $\tau=\left(\mathfrak{x}-\mathfrak{r}_{0}\right)^{2}-\left(1 / c^{2}\right)\left(z-z_{0}\right)$. Since

$$
L\left(z-z_{0}\right)=L z=\left[\begin{array}{lll}
\frac{1}{2 z}|\nabla \mathfrak{x}|^{2} & \text { on } & \mathscr{B}_{0}, \\
0 & \text { on } & \mathscr{T},
\end{array}\right.
$$

we infer that

$$
L \tau \geqq 2|\nabla \mathfrak{x}|^{2} \text { on } \mathscr{T}
$$

and that

$$
L \tau \geqq \frac{1}{2^{c}}\left(2 c^{2}-\frac{1}{z}\right)|\nabla \mathfrak{r}|^{2} \quad \text { on } \quad \mathscr{B}_{0} .
$$

Thus we obtain

LeMmA 4. If $0<\varepsilon \leqq z$, and if $c^{2} \geqq 1 / 2 \varepsilon$, it follows that

$$
L\left\{\left(\mathfrak{x}-\mathfrak{x}_{0}\right)^{2}-\frac{1}{c^{2}}\left(z-z_{0}\right)\right\} \geqq 0 \text { a.e. on } \mathscr{B} \text {. }
$$

To be somewhat more systematic we shall investigate for which $C^{2}$-functions $\alpha=\alpha(z)$ we can prove that

$$
L \sigma \geqq 0 \text { a.e. on } \mathscr{B} \text {, }
$$

where

$$
\sigma(u, v)=\left(\mathfrak{x}(u, v)-\mathfrak{x}_{0}\right)^{2}-\alpha(z(u, v)) .
$$

Because of the identity

$$
\Delta \alpha(z)=\alpha^{\prime \prime}(z)|\nabla z|^{2}+\alpha^{\prime}(z) \Delta z
$$

and of the Euler equations

$$
L z=\frac{1}{2 z}|\nabla \mathfrak{c}|^{2} \text { on } \mathscr{B}, \quad L z=0 \text { on } \mathscr{T}, \quad \Lambda \equiv 0 \text { on } \mathscr{T},
$$


we find that

$$
\begin{gathered}
\Delta \alpha(z)=\alpha^{\prime \prime}(z)|\nabla z|^{2}-\alpha^{\prime}(z) \Lambda z+\frac{1}{2 z} \alpha^{\prime}(z)|\nabla x y|^{2} \text { on } \mathscr{B}_{0}, \\
\Delta \alpha(z)=0 \text { a.e. } \mathscr{T} .
\end{gathered}
$$

Therefore,

$$
L \sigma \geqq 2|\nabla \mathfrak{r}|^{2} \geqq 0 \quad \text { a.e. on } \mathscr{J} \text {. }
$$

Since

$$
\Lambda \alpha(z)=\alpha^{\prime}(z) \Lambda z
$$

we infer that

$$
L \alpha(z)=\alpha^{\prime \prime}(z)|\nabla z|^{2}+\frac{\alpha^{\prime}(z)}{2 z}|\nabla \mathfrak{r}|^{2} \quad \text { on } \quad \mathscr{B} .
$$

Therefore,

$$
L \sigma=\left[2-\frac{\alpha^{\prime}(z)}{2 z}\right]|\nabla \mathfrak{r}|^{2}-\left\{\alpha^{\prime \prime}(z)+\frac{\alpha^{\prime}(z)}{2 z}\right\}|\nabla z|^{2} \quad \text { on } \quad \mathscr{B}_{0} .
$$

To obtain $L \sigma \geqq 0$, we must assume

$$
2-\frac{\alpha^{\prime}(z)}{2 z} \geqq 0
$$

Then,

$$
L \sigma \geqq\left[2-\frac{\alpha^{\prime}(z)}{z}-\alpha^{\prime \prime}(z)\right]|\nabla z|^{2} \quad \text { on } \mathscr{B} .
$$

The optimal choice is

$$
2-\frac{\alpha^{\prime}(z)}{z}-\alpha^{\prime \prime}(z)=0
$$

which is equivalent to

$$
\frac{d}{d z}\left\{z \frac{d}{d z} \alpha(z)\right\}=2 z
$$

The general solution $\alpha(z)$ of (4.24) is given by

$$
\alpha(z)=\frac{z^{2}}{2}+c_{1} \log z-c_{2}
$$

where $c_{1}$ and $c_{2}$ are arbitrary constants. However, this solution has to satisfy (4.22) which amounts to 


$$
3 z^{2} \geqq c_{1} .
$$

Since $z \geqq \varepsilon>0$, this is certainly satisfied if

$$
c_{1} \leqq 3 \varepsilon^{2} \text {. }
$$

This leaves us with three kinds of solutions of (4.23) which are admissible:

$$
\begin{gathered}
c_{1}=0: \alpha(z)=\frac{z^{2}}{2}-c_{2}, \quad z>0 . \\
0<c_{1} \leqq 3 \varepsilon^{2}: \alpha(z)=\frac{z^{2}}{2}+c_{1} \log z-c_{2}, \quad z \geqq \varepsilon . \\
c_{1}<0: \alpha(z)=\frac{z^{2}}{2}+c_{1} \log z-c_{2}, \quad z>0 .
\end{gathered}
$$

The solution (4.26) with $c_{2}=0$ leads to the composition used in Lemma 3 with $c=\sqrt{2}, z_{0}=0$.

Let us choose an arbitrary number $z_{0} \geqq 0$. A straightforward use of (4.26-4.28) yields the following result:

LEMMA 5. We have

$$
L\left\{\left(\mathfrak{r}-\mathfrak{r}_{0}\right)^{2}-\alpha_{j}(z)\right\} \geqq 0 \quad \text { on } \quad \mathscr{B}_{0}
$$

for

$$
\begin{array}{ll}
\alpha_{1}(z)=\frac{1}{2}\left(z^{2}-z_{0}^{2}\right), & z>0, \\
\alpha_{2}(z)=\frac{1}{2}\left(z^{2}-z_{0}^{2}\right)+3 \varepsilon^{2} \log \left(\frac{z}{z_{0}}\right), & z \geqq \varepsilon>0, \\
\alpha_{3}(z)=\frac{z^{2}}{2}-c \log z+\frac{c}{2}\left\{\log c-\frac{1}{2}-\log 2\right\}, & z>0, \quad c>0 .
\end{array}
$$

The function $\alpha_{2}(z)$ increases monotonically on $z>0$ and vanishes for $z=z_{0}$ if we suppose that $z_{0}>0$.

The hypersurface

$$
F=\left\{(x, y, z):\left(\mathfrak{x}-\mathfrak{r}_{0}\right)^{2}-\alpha_{2}(z)=0, z>0\right\}
$$

meets the parallel to the z-axis through $\mathfrak{x}_{0}=\left(x_{0}, y_{0}, z_{0}\right)$ exactly in $\mathfrak{x}_{0}$. For all points $x$ of $F$ different from $x_{0}$ we have $z>z_{0}$. The function $\alpha_{3}(z)$ vanishes for $z=z^{*}$, where

$$
z^{*}=\sqrt{\frac{c}{2}} .
$$


Moreover, $\alpha_{8}(z)$ increases for $z \geqq z^{*}$, and it decreases for $0<z \leqq z^{*}$. The hypersurface

$$
F^{*}=\left\{(x, y, z):\left(\mathfrak{x}-\mathfrak{r}_{0}\right)^{2}-\alpha_{3}(z)=0, z>0\right\}
$$

meets the parallel to the z-axis through $\mathfrak{x}_{0}=\left(x_{0}, y_{0}, z_{0}\right)$ exactly in $x_{0}^{*}=\left(x_{0}, y_{0}, z^{*}\right)$, since $\alpha_{3}(z)>\alpha_{3}\left(z^{*}\right)$ for $z>0, z \neq z^{*}$.

Now we use the various subsolutions of $L$ obtained in the Lemmata 2-5 to derive further inclusion theorems. As before, we shall assume for the rest of this section that $\mathfrak{x}(u, v)$ is a solution of the minimum problem $\mathfrak{P}(\Gamma)$ with the obstacle condition (4.2). The existence of such solutions for boundary curves $\Gamma$ contained in $\{z>\varepsilon\}, \varepsilon>0$, has been established in $\S 3$, together with their regularity properties. We proceed by applying Lemma 1 to the subsolutions of $L$ which have been discussed. Note, that each solution $\mathfrak{x}(u, v)=\left(x(u, v), y(u, v) h_{\Gamma} z(u, v)\right)$ of $\mathfrak{P}(\Gamma)$ satisfies

$$
\varepsilon \leqq z(u, v) \leqq h_{\Gamma} \text { for all }(u, v) \in \mathscr{B},
$$

by virtue of Theorem 4 , where $h_{\Gamma}$ is the maximal z-component of $\Gamma$ defined by (4.14).

THEOREM 5. Let $\mathscr{E}_{R}^{c}\left(\mathfrak{x}_{0}\right)$ denote the solid ellipsoid defined by

$$
\left(x-x_{0}\right)^{2}+\left(y-y_{0}\right)^{2}+\frac{1}{c^{2}}\left(z-z_{0}\right)^{2} \leqq R^{2}
$$

with center $x_{0}=\left(x_{0}, y_{0}, z_{0}\right)$, and with the semi-axes $R, R, c R$. Suppose that $z_{0}>0, \Gamma \subset \mathscr{E}_{R}^{c}\left(\mathfrak{\varepsilon}_{0}\right)$, and that

$$
\frac{z_{0}}{\varepsilon} \leqq \min \left\{2 c^{2}+1, c^{2}+2\right\}
$$

Then,

$$
\mathfrak{r}(\mathscr{B}) \subset \operatorname{int} \mathscr{E}_{R}^{c}\left(\mathfrak{x}_{0}\right)
$$

Assume now, that the interior of $\mathscr{E}_{R}^{c}\left(\mathfrak{x}_{0}\right)$ is contained in the open half space $\{z>\varepsilon\}$ which can always be achieved by appropriate choice of the parameters $\varepsilon, c, z_{0}, R$. Then

$$
z(u, v)>\varepsilon \text { on } \mathscr{B},
$$

and the set of coincidence $\mathscr{T}$ is empty. Therefore, $\mathfrak{x}(u, v)$ is real analytic in $\mathscr{B}$, and it satisfies the Euler equations and the conformality relations 


$$
\begin{aligned}
& L x=0 \\
& L y=0, \\
& L z=\frac{1}{2 z}|\nabla \mathfrak{x}|^{2} \quad \quad \mathfrak{x}_{u}^{2}=\mathfrak{x}_{v}^{2}, \quad \mathfrak{x}_{u} \cdot \mathfrak{x}_{v}=0
\end{aligned}
$$

on $\mathscr{B}$, where

$$
L=\Delta+\Lambda, \quad \Lambda=\frac{z_{u}}{z} \partial_{u}+\frac{z_{v}}{z} \partial_{v} .
$$

CoRollaRY 1. In particular, we can choose $z_{0}=h_{\Gamma}$ and $\varepsilon=h_{\Gamma} / 3$. Then, $z_{0} / \varepsilon=3$, and the choice $c=1$ is admissible for the parameter c.

Hence Theorem 5 applies to the ball

$$
B_{R}\left(\mathfrak{x}_{0}\right)=\left\{\mathfrak{x}:\left|\mathfrak{x}-\mathfrak{x}_{0}\right| \leqq R\right\}=\mathscr{E}_{R}^{1}\left(\mathfrak{x}_{0}\right) .
$$

Every $R>0$ is admissible but a very good choice will be

$$
R=\frac{2}{3} h_{\Gamma}
$$

This is the largest radius such that int $B_{R}\left(\mathfrak{X}_{0}\right) \subset\{z>\varepsilon\}$, whence $\mathscr{T}$ is empty, and $\mathfrak{r}(u, v)$ satisfies $(4.29)$ on $\mathscr{B}$.

Theorem 6. Let $\mathscr{C}_{c}\left(x_{0}\right)$ denote the solid cone

$$
\left(x-x_{0}\right)^{2}+\left(y-y_{0}\right)^{2}-\frac{1}{c^{2}}\left(z-z_{0}\right)^{2} \leqq 0
$$

with vertex $\mathfrak{x}_{0}=\left(x_{0}, y_{0}, z_{0}\right)$, and let $\mathscr{C}_{c}^{+}\left(\mathfrak{x}_{0}\right)$ denote its upper part $\mathscr{C}_{c}\left(\mathfrak{x}_{0}\right) \cap\left\{z \geqq z_{0}\right\}$. Suppose that $z_{0}>0, c \geqq 1, \Gamma \subset \mathscr{C}_{c}^{+}\left(\mathfrak{x}_{0}\right)$, and that

$$
c^{2}-2+\frac{z_{0}}{h_{\Gamma}} \geqq 0
$$

Then,

$$
\mathfrak{x}(\mathscr{B}) \subset \operatorname{int} \mathscr{C}_{c}^{+}\left(x_{0}\right) \text {. }
$$

If, in addition, int $\mathscr{C}_{\mathrm{c}}^{+}\left(\mathfrak{x}_{0}\right) \subset\{z>\varepsilon\}$, then

$$
z(u, v)>\varepsilon \text { on } \mathscr{B},
$$

and the set of coincidence $\mathscr{T}$ is empty. Therefore, $x(u, v)$ is real analytic in $\mathscr{B}$, and it satisfies (4.29) on $\mathscr{B}$.

Proof. The maximum principle implies that $\mathfrak{x}(\mathscr{B}) \subset \mathscr{C}_{c}\left(x_{0}\right)$. Yet, 
$\mathfrak{x}(\mathscr{S})$ could be partially contained in the lower part

$$
\mathscr{C}_{c}^{-}\left(\mathfrak{r}_{0}\right)=\mathscr{C}_{c}\left(x_{0}\right) \cap\left\{z \leqq z_{0}\right\}
$$

of the cone $\mathscr{C}_{c}\left(\mathfrak{x}_{0}\right)$. This will be excluded by the following reasoning: By virtue of the regularity results mentioned in $\S 3$ we may apply the - by now standard - Hartman/Wintner technique to obtain an asymptotic expansion of $\mathfrak{r}(u, v)$ around branch points which is of the same form as in Heinz-Hildebrandt [11]. This implies that $x(u, v)$ possesses a tangent plane even in branch points. For this reason, $\mathfrak{x}(u, v)$ cannot pass through the vertex $\mathfrak{x}_{0}$ of the cone $\mathscr{C}_{c}\left(\mathfrak{x}_{0}\right)$ whence either $\mathfrak{x}(\mathscr{B}) \subset \mathscr{C}_{c}^{+}\left(\mathfrak{x}_{0}\right)$ or $\mathfrak{x}(\mathscr{B}) \subset \mathscr{C}_{c}^{-}\left(\mathfrak{x}_{0}\right)$ must hold. Since $I \subset \mathscr{C}_{c}^{+}\left(\mathfrak{x}_{0}\right)$ we infer that $r(\mathscr{B}) \subset \mathscr{C}_{c}^{+}\left(\mathfrak{x}_{0}\right)$.

COROLLARY 2. In particular, $c \geqq \sqrt{\overline{2}}$ is always admissible whatever the value of $\varepsilon, z_{0}$, and $h_{\Gamma}$ may be. Choosing $\varepsilon=z_{0}=$ $h_{\Gamma}-R, c=\sqrt{2}, \Gamma \subset \mathscr{C}_{\sqrt{2}}^{+}\left(\mathfrak{r}_{0}\right), \mathfrak{x}_{0}=\left(x_{0}, y_{0}, \varepsilon\right)$, we infer that

$$
x(\mathscr{B}) \subset \operatorname{int} \in+\frac{1}{2}\left(x_{0}\right)
$$

whence

$$
z(u, v)>\varepsilon \text { for all }(u, v) \in \mathscr{B} .
$$

Thus $\mathscr{T}$ is empty, and $\mathfrak{r}(u, v)$ satisfies (4.29) on $\mathscr{B}$.

REMARK. The results of $\S 5$, and, in particular, Theorem 11, will furnish an improvement of Corollary 2.

Theorem 7. Let $\mathscr{P}_{c}\left(\mathrm{x}_{0}\right)$ denote the solid paraboloid

$$
\left(x-x_{0}\right)^{2}+\left(y-y_{0}\right)^{2}-\frac{1}{c^{2}}\left(z-z_{0}\right)<0
$$

where

$$
c^{2} \geqq \frac{1}{2 \varepsilon}
$$

and $\Gamma \subset \mathscr{P}_{c}\left(\mathfrak{X}_{0}\right)$. Then,

$$
\mathfrak{x}(\mathscr{B}) \subset \operatorname{int} \mathscr{P}_{c}\left(\mathfrak{x}_{0}\right)
$$

Moreover, $\mathscr{T}$ is empty and $\mathfrak{r}(u, v)$ satisfies (4.19) if int $\mathscr{P}_{c}\left(\mathfrak{x}_{0}\right) \subset$ $\{z>\varepsilon\}$.

Corollary 3. Determine $R>0$ by $h_{\Gamma}=3 R / 2$, and let $z_{0}=\varepsilon=$ $R / 2, c=1 / \sqrt{R}, \Gamma \subset \mathscr{P}_{1 / \sqrt{R}}\left(x_{0}\right)$. Then 


$$
\mathfrak{r}(\mathscr{B}) \subset \operatorname{int} \mathscr{P}_{1 / \sqrt{R}}\left(\mathfrak{x}_{0}\right) \subset\{z>\varepsilon\}
$$

whence $\mathfrak{x}(u, v)$ satisfies (4.29).

THEOREM 8. Let $z_{0}>0$, and denote by $\mathscr{L}_{\varepsilon}\left(x_{0}\right)$ the set

$$
\left\{(x, y, z):\left(x-x_{0}\right)^{2}+\left(y-y_{0}\right)^{2} \leqq \frac{1}{2}\left(z^{2}-z_{0}^{2}\right)+3 \varepsilon^{2} \log \left(\frac{z}{z_{0}}\right)\right\} .
$$

Suppose that $\Gamma \subset \mathscr{L}_{\varepsilon}\left(\mathfrak{x}_{0}\right)$. Then

$$
\mathfrak{x}(\mathscr{B}) \subset \operatorname{int} \mathscr{L}_{\varepsilon}\left(\mathfrak{x}_{00}\right)
$$

Furthermore, $\mathscr{T}$ is void, and $\mathfrak{x}(u, v)$ satisfies (4.29) if int $\mathscr{C}_{\varepsilon}\left(\mathfrak{x}_{0}\right) \subset$ $\{z>\varepsilon\}$.

THEOREM 9. Let $c>0, z^{*}=\sqrt{c / 2}, x_{0}^{*}=\left(x_{0}, y_{0}, z^{*}\right)$, and

$$
\begin{aligned}
\mathscr{N}_{c}\left(x_{0}^{*}\right) & =\left\{(x, y, z):\left(x-x_{0}\right)^{2}+\left(y-y_{0}\right)^{2}\right. \\
& \left.\leqq \frac{z^{2}}{2}-c \log z+\frac{c}{2}\left[\log c-\frac{1}{2}-\log 2\right]\right\} .
\end{aligned}
$$

Suppose that $\Gamma$ is contained in the upper part

$$
\mathscr{N}_{c}^{+}\left(\mathfrak{\complement}_{0}^{*}\right)=\mathscr{N}_{c}\left(\mathfrak{x}_{0}^{*}\right) \cap\left\{z \geqq z^{*}\right\}
$$

Then,

$$
\mathfrak{x}(\mathscr{B}) \subset \operatorname{int} \mathscr{N}_{c}^{+}\left(\mathfrak{x}_{00}^{*}\right)
$$

Moreover, $\mathscr{T}$ is empty, and $\mathfrak{x}(u, v)$ satisfies (4.29) if int $\mathscr{N}_{c}^{+}\left(\mathfrak{K}_{0}^{*}\right) \subset$ $\{z>\varepsilon\}$.

Remark concerning the proof. The same reasoning as in the proof of Theorem 6 applies. Because of the asymptotic expansion of $\mathfrak{r}(u, v)$ in the neighborhood of regular or branch points, $\mathfrak{x}(u, v)$ cannot fall below the "vertex" $x_{0.0}^{*}$.

Further maximum principles can be obtained by Hildebrandt [12], Theorem 8 , where $\mathfrak{x}(u, v)$ can either be interpreted as minimal surface in the Riemannian manifold $\boldsymbol{R}_{+}^{3}$ and $d s^{2}=z\left\{d x^{2}+d y^{2}+d z^{2}\right\}$, $\boldsymbol{R}_{+}^{3}=\boldsymbol{R}^{3} \cap\{z>0\}$, or as $\mathscr{H}$-surface in the euclidean space $E^{3}$, $\mathscr{C}$ defined by (2.13). This technique yields, for instance, the following result the proof of which will be omitted.

THEOREM 10. Let $z_{0}>0, \hat{\delta}>0, \Gamma \subset \mathscr{S}_{{ }_{-}^{+} 2} 2\left(\mathfrak{x}_{0}\right)$ where

$$
\mathscr{S}_{-\hat{\delta}^{2}}^{+}\left(\mathfrak{x}_{0}\right)=\left\{\left(\mathfrak{x}-\mathfrak{x}_{0}\right)^{2} \leqq \frac{1}{c^{2}}\left(z-z_{0}\right)^{2}-\delta^{2}\right\} \cap\left\{z \geqq z_{0}\right\} .
$$


Let $c>0$ be a real parameter such that

$$
\frac{1}{c^{2}}\left(1+\frac{1}{c^{2}}\right)\left(z-z_{0}\right)^{2}-\frac{z^{2}}{4} \leqq \delta^{2} \quad \text { for } \quad c \delta \leqq z \leqq h_{\digamma} .
$$

Suppose that $\mathfrak{r}(u, v)$ is a solution of the Plateau problem

$$
\begin{aligned}
\int_{.0} z|\nabla \mathfrak{x}|^{2} d u d v & \longrightarrow \min , \quad \mathfrak{r}(\mathscr{B}) \subset \mathscr{S}_{-{ }^{2}}^{+}\left(\mathfrak{E}_{0}\right), \\
\mathfrak{x}: \partial \mathscr{B} & \longrightarrow I \text { monotonically } .
\end{aligned}
$$

Then,

$$
\mathfrak{x}(\mathscr{B}) \subset \operatorname{int} \mathscr{S}_{-\delta^{2}}^{+}\left(\mathfrak{x}_{0}\right),
$$

$\mathscr{T}$ is empty, and $\mathfrak{r}(u, v)$ satisfies (4.29).

(ii) Another maximum principle (functioning already for harmonic maps with obstacles) is contained in Hildebrandt-Kaul [15], Lemma 9, Theorems 8 and 9, pp. 217-221. Here we consider $\mathfrak{x}(u, v)$ as minimal surface with respect to the metric $d \mathrm{~s}^{2}=z \cdot\left\{d x^{2}+d y^{2}+\right.$ $\left.d z^{2}\right\}$ which has been extensively studied. The geodesics are parabolae, and the geodesic balls (used in [15] for the maximum principle) are explicitely determined, cf. [10], and, in particular Darboux [7]. However, we have not worked out details.

REMARK. In view of Gulliver's theorem in [9], a solution of the Plateau problem has no interior branch points if it does not touch $\{z=\varepsilon\}$, the boundary of the obstacle.

Two further methods to obtain inclusion theorems will be presented in $\S \S 5$ and 6 . The first one is a refinement of the technique used in this section, while the second one is based on an application of some isoperimetric inequality.

5. Inclusion theorem for the solutions of $\mathfrak{P}(\Gamma)$ when $J(\mathfrak{x})=$ $\int z|\nabla \times|^{2} d u d v$, and when $\mathscr{K}$ is the upper part of the solid hyperboloid $\left\{x: z^{2} \geqq\left(x-x_{0}\right)^{2}+\left(y-y_{0}\right)^{2}+\varepsilon^{2}\right\}$. In this section, we consider once more the variational problem $\mathfrak{B}(\Gamma)$ for the integral

$$
J(\mathfrak{x})=\int_{\sigma^{\prime}} z|\nabla \mathfrak{x}|^{2} d u d v
$$

which, for conformal parameters $u, v$ of $x$, agrees with

$$
J^{*}(\mathfrak{x})=2 \int_{\mathscr{B}} z\left|\mathfrak{x}_{u} \times \mathfrak{x}_{v}\right| d u d v .
$$

Let $\mathfrak{r}_{0}=\left(x_{0}, y_{0}, 0\right)$ be a fixed point in $\boldsymbol{R}^{3}$, and denote by $\mathscr{C}=\mathscr{C}\left(\mathfrak{x}_{0}\right)$ 
the cone

$$
\left\{x: z^{2}=\left(x-x_{0}\right)^{2}+\left(y-y_{0}\right)^{2}=\left(\mathfrak{r}-\mathfrak{r}_{0}\right)^{2}\right\} .
$$

It turns out that each Gauss representation $\mathfrak{x}=\mathfrak{x}(u, v)$ of $\mathscr{C}^{\prime}=\mathscr{C}-$ $\left\{\mathfrak{r}_{0}\right\}$ furnishes a solution of the Euler equation belonging to (5.2). This can easily be checked by proving that

$$
u(x, y)= \pm \sqrt{\left(x-x_{0}\right)^{2}+\left(y-y_{0}\right)^{2}}= \pm\left|\mathfrak{x}-\mathfrak{x}_{0}\right|, \quad \mathfrak{x} \neq \mathfrak{x}_{0},
$$

is a solution of

$$
\frac{\partial}{\partial x}\left\{u \frac{u_{x}}{\sqrt{1+|\nabla u|^{2}}}\right\}+\frac{\partial}{\partial y}\left\{u \frac{u_{y}}{\sqrt{1+|\nabla u|^{2}}}\right\}=\sqrt{1+|\nabla u|^{2}}
$$

which is the Euler equation of the integral

$$
\int u \sqrt{1+|\nabla u|^{2}} d x d y \text {. }
$$

However, this integral is the nonparametric counterpart of (5.2).

This observation suggests that the upper part of the cone $\left\{\mathrm{r}: z^{2} \geqq\left|\mathfrak{r}-\mathfrak{x}_{0}\right|^{2}\right\}$ forms a domain of inclusion for the variational problem $\mathfrak{P}(\Gamma)$. This is in fact true. Since the cone has an unpleasant singularity in its vertex $\mathfrak{r}_{0}$ we perfer to look at the upper sheet

$$
S_{-\varepsilon^{2}}^{+}=S_{-\varepsilon^{2}} \cap\{z \geqq 0\}
$$

of the two-sheeted solid hyperboloid

$$
S_{-\varepsilon^{2}}=\left\{\left(x-x_{0}\right)^{2}+\left(y-y_{0}\right)^{2}+\varepsilon^{2} \leqq z^{2}\right\}, \quad \varepsilon>0 .
$$

The reasoning is similar to the one used in $\S 4$ but more elaborate. We begin with two observations which can be considered as a refinement of the inequality

$$
|\nabla x|^{2}+|\nabla y|^{2} \leqq|\nabla z|^{2}
$$

following from the conformality relations

$$
\mathfrak{x}_{u}^{2}=\mathfrak{x}_{v}^{2}
$$

and

$$
\mathfrak{x}_{u} \cdot \mathfrak{x}_{v}=0 \text {. }
$$

Lemma 6. Let $\mathfrak{r}(u, v)=(x(u, v), y(u, v), z(u, v))$ be a function of class $C^{1}\left(\mathscr{B}, \boldsymbol{R}^{3}\right)$ which satisfies (5.3) and (5.4). Denote $\mathfrak{x}_{u} \times \mathrm{x}_{v}$ by $(\xi, \eta, \zeta)$. Then, the following relations hold: 


$$
\begin{aligned}
& 4 \xi^{2}=\left\{|\nabla y|^{2}+|\nabla z|^{2}\right\}^{2}-|\nabla x|^{4} \\
& 4 \eta^{2}=\left\{|\nabla x|^{2}+|\nabla z|^{2}\right\}^{2}-|\nabla y|^{4} \\
& 4 \zeta^{2}=\left\{|\nabla x|^{2}+|\nabla y|^{2}\right\}^{2}-|\nabla z|^{4} .
\end{aligned}
$$

Moreover,

$$
|\nabla z|^{2}=0 \text { if } \xi^{2}+\eta^{2}=0
$$

and

$$
|\nabla z|^{2}=\frac{1}{2 c^{2}+1}\left\{|\nabla x|^{2}+|\nabla y|^{2}\right\} \quad \text { if } \quad \xi^{2}+\eta^{2}>0
$$

and

$$
c^{2}=\frac{\zeta^{2}}{\xi^{2}+\eta^{2}}
$$

Proof. The relations (5.6) and (5.7) follow from (5.5) by a straightforward computation. Moreover, the second and the third identity of (5.5) can be obtained from the first one. Thus it suffices to prove that

$$
4 \xi^{2}=\left\{|\nabla y|^{2}+|\nabla z|^{2}\right\}^{2}-|\nabla x|^{4}
$$

By (5.4), we get

$$
\xi^{2}=\left(y_{u} z_{v}-y_{v} z_{u}\right)^{2}=\left(y_{u}^{2}+z_{u}^{2}\right)\left(y_{v}^{2}+z_{v}^{2}\right)=x_{u}^{2} x_{v}^{2} .
$$

On the other hand, by (5.3),

$$
\begin{aligned}
& \left\{|\nabla y|^{2}+|\nabla z|^{2}\right\}^{2}-|\nabla x|^{4} \\
& \quad=2\left(y_{u}^{2}+z_{u}^{2}\right)\left(y_{v}^{2}+z_{v}^{2}\right)+\left(y_{u}^{2}+z_{u}^{2}\right)^{2}+\left(y_{v}^{2}+z_{v}^{2}\right)^{2}-\left(x_{u}^{2}+x_{v}^{2}\right)^{2} \\
& \quad=2\left(y_{u}^{2}+z_{u}^{2}\right)\left(y_{v}^{2}+z_{v}^{2}\right)+\left(y_{v}^{2}+z_{v}^{2}+x_{v}^{2}-x_{u}^{2}\right)^{2}+\left(y_{v}^{2}+z_{v}^{2}\right)^{2}-\left(x_{u}^{2}+x_{v}^{2}\right)^{2} \\
& \quad=2\left(y_{v}^{2}+z_{v}^{2}\right)\left\{\left(y_{u}^{2}+z_{u}^{2}\right)+\left(y_{v}^{2}+z_{v}^{2}\right)+\left(x_{v}^{2}-x_{u}^{2}\right)\right\}-4 x_{u}^{2} x_{v}^{2} \\
& \quad=4\left(y_{u}^{2}+z_{u}^{2}\right)\left(y_{v}^{2}+z_{v}^{2}\right)-4 x_{u}^{2} x_{v}^{2} .
\end{aligned}
$$

Thus, the lemma is proved.

THeOREM 11. Let $\mathfrak{x}(u, v)$ be a solution of Plateau problem

$$
\int_{\mathscr{B}} z|\nabla \mathfrak{x}|^{2} d u d v \longrightarrow \min , \quad \mathfrak{x}(\mathscr{B}) \subset S_{-\varepsilon^{2}}^{+}=\mathscr{K}, \quad \mathfrak{r}: \partial \mathscr{B} \longrightarrow \Gamma
$$

where

$$
S_{-\varepsilon^{2}}^{+}=\left\{x:\left|\mathfrak{x}-\mathfrak{x}_{0}\right|^{2}+\varepsilon^{2} \leqq z^{2} \text { and } z \geqq 0\right\}
$$

for some $\mathfrak{r}_{0}$ and some $\varepsilon>0$. Suppose $\Gamma$ is contained in the interior 
of $S_{-\varepsilon^{2}}^{+}$. Then, $\mathfrak{x}(\mathscr{B}) \subset \operatorname{int} S_{-\varepsilon^{2}}^{+}$. In particular, $\mathfrak{r}(u, v)$ is a solution of the Euler equation (4.29).

Proof. Without loss of generality, we may assume that $x_{0}=$ $y_{0}=0$. Note that $x(u, v)$ is of class $H_{2}^{2}\left(\mathscr{B}, \boldsymbol{R}^{3}\right) \cap C^{1, \alpha}\left(\mathscr{\mathscr { B }}, \boldsymbol{R}^{3}\right) 0<\alpha<1$. Let $\sigma(u, v)$ be defined by

$$
\sigma(u, v)=x^{2}(u, v)+y^{2}(u, v)-z^{2}(u, v)=\mathfrak{x}^{2}(u, v)-z^{2}(u, v) .
$$

In virtue of Lemma 1 , the theorem is proved if we can find functions $a(u, v), b(u, v)$ of class $L^{\infty}(\mathscr{B}, \boldsymbol{R})$ such that

$$
L \sigma \geqq 0 \text { a.e. on } \mathscr{B}
$$

where

$$
L=\frac{1}{2}\left(\Delta+a \frac{\partial}{\partial u}+b \frac{\partial}{\partial v}\right) .
$$

The construction of the functions $a$ and $b$ will be carried out in four steps.

First, we observe that

$$
\Delta \sigma=0 \text { a.e. on } \mathscr{Y}
$$

where

$$
\mathscr{T}=\left\{(u, v) \in \mathscr{B}: \mathfrak{x}(u, v) \in \partial S_{-\varepsilon^{2}}^{+}\right\}
$$

denotes the "set of coincidence" of the solution vector $x(u, v)$. The boundary $\partial S_{-\varepsilon^{2}}^{+}$of the set of inclusion is nothing but the graph of the function

$$
z=U(x, y)=\sqrt{x^{2}+y^{2}+\varepsilon^{2}}
$$

in $\boldsymbol{R}^{3}$. In virtue of $\sigma \in H_{2}^{2}(\mathscr{B}, \boldsymbol{R})$ and of $\sigma(u, v)=0$ on $\mathscr{T},(5.11)$ is an immediate consequence of a well known result due to Morrey, cf. [19], p. 69 .

On $\mathscr{B}-\mathscr{T}, \mathfrak{x}(u, v)$ is real analytic and satisfies the Euler equations

$$
\Delta \mathfrak{x}=\frac{\zeta}{z} \mathfrak{N}=\frac{\zeta}{z W} \mathfrak{x}_{u} \times \mathfrak{x}_{v}, \quad W=\left|\mathfrak{x}_{u} \times \mathfrak{r}_{v}\right|
$$

Choosing

$$
L=\frac{1}{2}\left\{\Delta+a \frac{\partial}{\partial u}+b \frac{\partial}{\partial v}\right\} .
$$


where $a(u, v)$ and $b(u, v)$ are to be determined later on, we arrive at

$$
L \sigma=|\nabla \mathfrak{r}|^{2}-|\nabla \boldsymbol{z}|^{2}+(x, y,-z) \cdot\left\{a \mathfrak{x}_{u}+b \mathfrak{r}_{v}+\frac{\zeta}{z} \mathfrak{l}\right\} .
$$

Since $\left\{\mathfrak{x}_{u} /\left|\mathfrak{x}_{u}\right|, \mathfrak{x}_{v} /\left|\mathfrak{x}_{v}\right|, \mathfrak{R}\right\}$ form an orthonormal system for each $(u, v) \in \mathscr{B}$, there exist uniquely determined numbers $\alpha(u, v), \beta(u, v)$, $\gamma(u, v)$ such that

$$
(x, y,-z)=\alpha \frac{\mathfrak{x}_{u}}{|\underline{x} u|}+\beta \frac{\mathfrak{r}_{v}}{|\underline{x} v|}+\gamma \mathscr{U}
$$

whence

$$
L \sigma=|\nabla \mathfrak{r}|^{2}-|\nabla z|^{2}+(\alpha \alpha+b \beta) \frac{1}{\sqrt{2}}|\nabla \mathfrak{x}|+\frac{\gamma \zeta}{z}
$$

and

$$
-z=\frac{\alpha z_{u}}{\left|\mathfrak{x}_{u}\right|}+\frac{\beta z_{v}}{\left|\mathfrak{x}_{v}\right|}+\frac{\gamma \zeta}{W}=\frac{\sqrt{2}}{|\nabla \mathfrak{x}|}\left(\alpha z_{u}+\beta z_{v}\right)+\frac{2}{|\nabla \mathfrak{x}|^{2}} \gamma \zeta .
$$

Therefore,

$$
\frac{\gamma \zeta}{z}=-\frac{|\nabla \mathfrak{r}|}{\sqrt{2}}\left\{\frac{|\nabla \mathfrak{r}|}{\sqrt{2}}+\frac{\alpha z_{u}}{z}+\frac{\beta z_{v}}{z}\right\}
$$

and

$$
L \sigma=\frac{1}{2}|\nabla \mathfrak{x}|^{2}-\frac{3}{2}|\nabla z|^{2}+\frac{|\nabla \mathfrak{x}|}{2}\left[\alpha\left(a-\frac{z_{u}}{z}\right)+\beta\left(b-\frac{z_{v}}{z}\right)\right] .
$$

We choose $a(u, v)$ and $b(u, v)$ in the form

$$
a=\frac{z_{u}}{z}+\sqrt{2} a_{0}|\nabla \mathfrak{x}|, \quad b=\frac{z_{v}}{z}+\sqrt{2} b_{0}|\nabla \mathfrak{x}|
$$

where $a_{0}(u, v), b_{0}(u, v)$ are to be determined later on. Then

$$
L \sigma=\frac{1}{2}|\nabla \mathfrak{r}|^{2}-\frac{3}{2}|\nabla z|^{2}+\left(a_{0} \alpha+b_{0} \beta\right)|\nabla \mathfrak{r}|^{2} .
$$

If $\xi^{2}+\eta^{2}=0$, we have $|\nabla z|^{2}=0$, on account of (5.6). Choosing

$$
a_{0}=b_{0}=0 \text { if } \xi^{2}+\eta^{2}=0,
$$

we find that

$$
L \sigma=\frac{1}{2}|\nabla \mathfrak{x}|^{2} \geqq 0 \quad \text { when } \quad \xi^{2}+\eta^{2}=0 .
$$

Now we consider the case $\xi^{2}+\eta^{2} \neq 0$. By (5.7), 


$$
|\nabla \mathfrak{r}|^{2}=\left(2 c^{2}+1\right)|\nabla z|^{2}, \quad \text { where } \quad c^{2}=\frac{\zeta^{2}}{\xi^{2}+\eta^{2}},
$$

and we infer from (5.15) that

$$
L \sigma=\left\{\left(c^{2}-1\right)+2\left(c^{2}+1\right)\left(a_{0} \alpha+b_{0} \beta\right)\right\}|\nabla z|^{2} \text { if } \xi^{2}+\eta^{2} \neq 0 .
$$

Choosing

$$
a_{0}=b_{0}=0 \text { if } \quad c \geqq 1,
$$

we obtain once more

$$
L \sigma=\left(c^{2}-1\right)|\nabla z|^{2} \geqq 0 \quad \text { for } \quad c \geqq 1 .
$$

The condition $c \geqq 1$ implies that $\mathfrak{R}$ lies inside the cone $\left\{z^{2} \geqq x^{2}+y^{2}\right\}$. Moreover, we claim that

$$
|\mathfrak{x}| \leqq \frac{\sqrt{2+2 c^{2}}}{1-c} \sqrt{\alpha^{2}+\beta^{2}} \text { if } 0 \leqq c<1 .
$$

Let us put off the proof of this inequality for the moment, and choose

$$
a_{0}=\frac{2}{\varepsilon} \frac{\alpha}{\sqrt{\alpha^{2}+\beta^{2}}}, \quad b_{0}=\frac{2}{\varepsilon} \frac{\beta}{\sqrt{\alpha^{2}+\beta^{2}}} \quad \text { if } \quad 0 \leqq c<1 .
$$

Then, we obtain from (5.18) that

$$
\begin{aligned}
L \sigma & =\left\{\left(c^{2}-1\right)+\frac{4}{\varepsilon}\left(c^{2}+1\right) \sqrt{a^{2}+\beta^{2}}\right\}|\nabla z|^{2} \\
& \geqq\left\{\left(c^{2}-1\right)+2 \sqrt{2} \sqrt{1+c^{2}} \frac{|\mathfrak{x}|}{\varepsilon}(1-c)\right\}|\nabla z|^{2} .
\end{aligned}
$$

Since $0 \leqq c<1$ and $|x| \geqq \varepsilon$, we infer that

$$
L \sigma \geqq\left\{\left(c^{2}-1\right)+2(1-c)\right\}|\nabla z|^{2} \geqq 0 \quad \text { if } \quad 0 \leqq c<1 .
$$

Note that $\mathfrak{x}$ is of class $C^{1}\left(\overline{\mathscr{B}}, \boldsymbol{R}^{3}\right)$. Choosing

$$
a=b=0 \text { on } \mathscr{Y} \text {, }
$$

we have $a, b \in L^{\infty}(\mathscr{B})$ taking (5.14), (5.16), (5.19), (5.22). and (5.24) into account, and

$$
L \sigma \geqq 0 \text { a.e. on } \mathscr{B}
$$

by virtue of (5.11), (5.17), (5.20), and (5.23).

It remains to prove (5.21) where

$$
\zeta^{2}=c^{2} \cdot\left\{\xi^{2}+\eta^{2}\right\}, \quad 0 \leqq c<1 .
$$


Since $(u, v) \in S_{-\varepsilon^{2}}^{+}$, we have also

$$
x^{2}+y^{2} \leqq z^{2},
$$

and

$$
\mathfrak{x}^{*}=(x, y,-z)=\alpha \frac{\mathfrak{x}_{u}}{\left|\mathfrak{x}_{u}\right|}+\beta \frac{\mathfrak{x}_{v}}{\left|\mathfrak{x}_{v}\right|}+\gamma \mathfrak{R} .
$$

Let $\varphi$ be the angle between $\mathfrak{r}^{*}$ and $\mathfrak{R}$. Then

$$
\sin \varphi=\frac{\sqrt{\alpha^{2}+\beta^{2}}}{\sqrt{\alpha^{2}+\beta^{2}+\gamma^{2}}}=\frac{\sqrt{\alpha^{2}+\beta^{2}}}{\left|\mathfrak{x}^{*}\right|}=\frac{\sqrt{\alpha^{2}+\beta^{2}}}{|\mathfrak{x}|} .
$$

Furthermore, let $\psi$ be the smallest angle between $\mathfrak{N}$ and the cone $\left\{x^{2}+y^{2}=z^{2}\right\}$. By virtue of (5.25), $\psi$ is simply the angle between the two lines $z=r$ and $z=c r$ in $(r, z)$-plane whence

$$
\sin \psi=\frac{1}{\sqrt{2}} \frac{1-c}{\sqrt{1+c^{2}}} .
$$

Finally, the relations (5.25) and (5.26) imply that

$$
\sin \psi \leqq \sin \varphi
$$

which, in turn, yields the desired estimate (5.21). Thus, the theorem is proved.

6. A further inclusion theorem based on an estimate due to Allard-Michael-Simon. The inclusion theorem of this section is based on the following

Lemma 7. Let $\mathscr{F}$ be a $C^{2}$-submanifold of $\boldsymbol{R}^{N}$ of dimension $n$ and codimension $p$, i.e., $N=n+p$. For an inner point $\xi$ of $\mathscr{F}$, and for $\rho \in \boldsymbol{R}^{+}$, we define

$$
m(\rho)=m_{\xi}(\rho)=\frac{1}{\rho^{n}} \int_{\substack{|x-\xi|<\rho \\ x \in G \mathscr{G}}} d \cdot \mathscr{X}(x),
$$

where d. $\mathscr{A}(x)$ denotes the n-dimensional area element of $\mathscr{F}$ at $x$. Suppose that, for some $\rho_{0}>0$, the boundary of the set $\mathscr{F}_{0}=\{x \in \mathscr{F}$ : $\left.\left|x-x_{0}\right| \leqq \rho\right\}$, considered as compact submanifold with boundary, is contained in the sphere $\left\{x \in \boldsymbol{R}^{N}:|x-\xi|=\rho_{0}\right\}$. Moreover, we assume that the absolute value of the mean curvature $\mathscr{C}$ of $\mathscr{F}$ is bounded. on $\mathscr{F}_{0}$ by some number $H \geqq 0$. Then we have

(i) $\lim _{\rho \rightarrow+0} m(\rho)=m(0)=\omega_{n}$,

where $\omega_{n}$ denotes the volume of the n-dimensional unit ball;

(ii) $-\frac{d}{d \rho}[m(\rho)] \leqq H m(\rho)$ for $0<\rho \leqq \rho_{0}$; 
(iii) $m(\rho) \geqq \omega_{n} \exp (-H \rho)$ for $0 \leqq \rho \leqq \rho_{0}$.

Proof. (i) is an obvious consequence of the manifold structure of $\mathscr{F}$ at $\xi$. (ii) is a very special case of an important Sobolev type inequality due to Michael and Simon [17]. A variant of this estimate was first derived by Allard [1]. (iii) follows immediately from (ii) by integration.

LEMMA 8. Suppose that the assumptions of Lemma 7 are satisfied, with the exception that $\mathscr{F}$ is not a $C^{2}$-submanifold of $\boldsymbol{R}^{N}$ but only a $C^{2}$-immersion into $\boldsymbol{R}^{N}$ of dimension $n$ and codimension p. Then, the total area $A\left(\mathscr{F}_{0}\right)$ of $\mathscr{F}_{0}$ can be estimated from below by

$$
A\left(\mathscr{F}_{0}\right) \geqq \rho_{0}^{n} \exp \left(-H \rho_{0}\right)
$$

Proof. If $\mathscr{F}_{0}$ is a submanifold of $R^{N}$ the assertion follows from (iii) of Lemma 7. If $\mathscr{F}_{0}$ is only immersed and $H=0$ the estimate can be obtained from [1]. If $H \geqq 0$ we can proceed as follows: If $\mathscr{F}_{0}$ is an immersion into $\boldsymbol{R}^{N}$, then it is also immersed into $\boldsymbol{R}^{N+q}$, $q>0$, if $\boldsymbol{R}^{N}$ is canonically embedded into $\boldsymbol{R}^{N+q}$. Clearly, the codimension of $\mathscr{F}_{0}$ is now $p+q$. It is elementary to approximate $\mathscr{F}_{0}$ in $\boldsymbol{R}^{N+q}$ by a sequence of $n$-dimensional submanifolds $\tilde{\mathscr{F}_{0}}$ with boundary on $\left\{X \in \boldsymbol{R}^{N+q}:|X-\Xi|=\rho_{0}\right\}, \Xi=(\xi, 0)$, such that the mean curvature of the $\tilde{\mathscr{F}}_{0}$ tends uniformly to the mean curvature of $\mathscr{F}_{0}$, as $\tilde{F}_{0}$ tends to $\mathscr{F}_{0}$, at least, if $q$ is sufficiently large. Lemma 7 , (iii) applies to each of the $\tilde{\mathscr{F}}_{0}$, and, by passing to the limit, we obtain (6.1).

Suppose now that $\Gamma$ is a closed regular Jordan curve $\boldsymbol{R}^{3}$ of class $C^{2},{ }^{\beta}, 0<\beta<1$, which is contained in the slab

$$
S\left(h_{1}, h\right)=\left\{(x, y, z) \in \boldsymbol{R}^{3}: h_{1}<z \leqq h\right\}
$$

where

$$
0<h_{1} \leqq h \text {. }
$$

Then we choose a number $\varepsilon>0$ such that

$$
h_{1}>5 \varepsilon \text {. }
$$

Moreover, let $\omega(z), z \in \boldsymbol{R}$, be a function of class $C^{2}$ such that

$$
\omega(z)=z \text { for } z \geqq \varepsilon .
$$

Approximation the Lipschitz function 


$$
\omega_{0}(z)= \begin{cases}\varepsilon & \text { for } z \leqq \varepsilon \\ z & \text { for } z>\varepsilon\end{cases}
$$

in an appropriate way by functions $\omega(z)$, we infer that, for every $\delta \in(0, \varepsilon / 2)$, there exists a $C^{2}$-function $\omega(z)$ satisfying $(6.4)$,

$$
\varepsilon / 2 \leqq \varepsilon-\delta \leqq \omega(z) \text { for all } z \in R
$$

and

$$
0 \leqq \frac{\omega^{\prime}(z)}{2 \omega(z)} \leqq \frac{1}{2 \varepsilon}+\delta \quad \text { for all } z \in R
$$

Consider now the Plateau problem

$$
\mathfrak{P}(\Gamma): \quad J \longrightarrow \min \text { on } \mathbb{S}(\Gamma)
$$

where

$$
J(\mathfrak{x})=\int_{\infty} \omega(z)|\nabla \mathfrak{x}|^{2} d u d v
$$

and where $\mathbb{S}(\Gamma)$ denotes the class of all surfaces in $H_{2}^{1}\left(\mathscr{B}, \boldsymbol{R}^{3}\right)$ which map $\partial \mathscr{B}$ continuously and in a weakly monotonic way onto $\Gamma$. By virtue of Theorems $1-3$, there exists a solution of $\mathfrak{P}(\Gamma)$; and each solution $\mathfrak{r}(u, v)$ of $\mathfrak{P}(\Gamma)$ is of class $C^{2}\left(\overline{\mathscr{B}}, \boldsymbol{R}^{3}\right)$ and satisfies

$$
\Delta \mathfrak{x}=\mathscr{H}(x, \mathfrak{R})\left[\mathfrak{x}_{u} \times \mathfrak{x}_{v}\right]
$$

and

$$
\mathfrak{x}_{u}^{2}=\mathfrak{x}_{v}^{2}, \quad x_{u} \cdot \mathfrak{x}_{v}=0
$$

on $\mathscr{B}$, where

$$
\mathscr{L}(\mathfrak{x}, \mathfrak{R})=\frac{\omega^{\prime}(z)}{2 \omega(z)} \cdot N^{3}, \quad \mathfrak{N}=\left(N^{1}, N^{2}, N^{3}\right) .
$$

On account of Gulliver's theorem [9], గֵ. $(u, v)$ possesses no branch points in $\mathscr{B}$. Therefore,

$$
\mathscr{F}=\{\mathfrak{x}(u, v):(u, v) \in \mathscr{B}\}
$$

is a $C^{2}$-immersion into $R^{3}$ of dimension $n=2$ and of codimension 1 , the mean curvature of which is bounded by

$$
|\mathscr{H}| \leqq H=\frac{1}{2 \varepsilon}+\delta
$$

THEOREM 12. Let $\mathfrak{r}=\mathfrak{r}(u, v)$ be a solution of the Plateau problem $\mathfrak{P}(\Gamma)$, and suppose that (6.2)-(6.6) are satisfied. Assume also that 
$\Gamma \subset S\left(h_{1}, h\right)$, and that

$$
A(\mathscr{F})=A\left(\mathfrak{x}_{0}\right)=\int_{\mathscr{O}}\left|\mathfrak{x}_{u} \times \mathfrak{x}_{v}\right| d u d v<2^{4} \pi e^{-2} \varepsilon^{2}
$$

holds, where $\mathscr{F}$ is defined by (6.8). Then, $\mathscr{F}$ is contained in the open half space $\{z>\varepsilon\}$. Hence $z(u, v)=\omega(z(u, v))$ on $\mathscr{B}$. Therefore, $\mathfrak{x}(u, v)$ is real analytic and satisfies (4.29) on $\mathscr{B}$.

Proof. Otherwise there is a point $x_{0}=\left(x_{0}, y_{0}, z_{0}\right) \in \mathscr{F}$ such that $z_{0}=\varepsilon$. On account of (6.6) and of $\Gamma \subset S\left(h_{1}, h\right)$ and $0<\varepsilon<5 \varepsilon \leqq h_{1}$, we can apply Lemma 8 with $\rho_{0}=h_{1}-\varepsilon$ and with $H=1 /(2 \varepsilon)+\delta$ thus obtaining

$$
A(\mathscr{F})>\pi \rho^{2} e^{-H \rho} \text { for } 0<\rho \leqq \rho_{0} \text {. }
$$

The function $\rho^{2} e^{-H \rho}$ achieves its maximum for $2-H \rho=0$, i.e., $\rho=2 / H=4 \varepsilon+\eta(\delta)$, where $\eta(\delta)>0$, and $\eta(\delta) \rightarrow+0$ as $\delta \rightarrow+0$. Hence, there is a $\delta_{0}>0$ such that

$$
\rho=\frac{2}{H}=4 \varepsilon+\eta(\delta) \leqq \rho_{0}=h_{1}-\varepsilon \text { for } 0<\delta<\delta_{0},
$$

whence

$$
A(\mathscr{F})>\pi(4 \varepsilon)^{2} e^{-2}
$$

But this is impossible, because of assumption (6.10). The theorem is proved.

Lemma 9. Let $\mathfrak{x}=\mathfrak{x}(u, v)$ be a solution of $\mathfrak{B}(\Gamma)$, and suppose that $\Gamma \subset S\left(h_{1}, h\right)$, and that (6.2)-(6.6) hold. Then

$$
A(x)<\frac{h}{\varepsilon-\delta} a(\Gamma)
$$

where $a(\Gamma)$ denotes the minimum of the area of all surfaces of the type of the disc which are bounded by $\Gamma$ (i.e., are of class $\mathbb{(}(\Gamma))$.

Proof. Let $D(x)$ be the standard Dirichlet integral of $x(u, v)$ :

$$
D(\mathfrak{x})=\int_{\mathscr{G}}|\nabla \mathfrak{x}|^{2} d u d v
$$

Then

$$
D(x)=2 A(x) .
$$

On account of (6.5), we obtain 


$$
(\varepsilon-\delta) D(\mathfrak{x})<J(\mathfrak{x}) .
$$

Let $\mathrm{f}(u, v),(u, v) \in \mathscr{B}$, be a minimal surface bounded by $\Gamma$ which is of minimal area. That is, $f \in \mathbb{C}(\Gamma)$,

$$
\Delta \mathfrak{f}=0, \quad \mathfrak{f}_{u}^{2}=\mathfrak{f}_{v}^{2}, \quad \mathfrak{f}_{u} \cdot \mathfrak{f}_{v}=0 \quad \text { in } \mathscr{B},
$$

and

$$
A(\mathfrak{f})=\frac{1}{2} D(\mathfrak{f})=a(\Gamma) .
$$

Then,

$$
J(\mathfrak{x}) \leqq J(\mathfrak{f}),
$$

because $x$ minimizes $J$ in $\mathbb{E}(\Gamma)$. Since $\Gamma \subset S\left(h_{1}, h\right)$ the maximum principle implies that $\mathrm{f}(B) \subset S\left(h_{1}, h\right)$ whence

$$
J(\mathfrak{f}) \leqq h D(\mathfrak{f}) .
$$

Combining these estimates, we arrive at (6.11).

Theorem 12 and Lemma 9 imply immediately

THEOREM 13. Let $\mathfrak{x}=\mathfrak{r}(u, v)$ be a solution of $\mathfrak{P}(\Gamma)$, and denote by $a(\Gamma)$ the minimum of the area of all disc type surfaces spanning $\Gamma$. Suppose that $\Gamma \subset S\left(h_{1}, h\right)$, and that (6.2)-(6.6) are satisfied as well as

$$
a(\Gamma) \leqq \frac{\varepsilon}{h} \pi\left(\frac{4 \varepsilon}{e}\right)^{2} .
$$

Then $r(\mathscr{B}) \subset\{z>\varepsilon\}, z(u, v)=\omega(z(u, v))$ on $\mathscr{P}, \mathfrak{x}(u, v)$ is real analytic on $\mathscr{B}$ and satisfies (4.29).

REMARK. A good choice of $\varepsilon$ would be $\varepsilon$ close to $h_{1} / 5$ in which case the condition (6.12) takes the form

$$
a(\Gamma)<\frac{\pi}{5} \cdot \frac{h_{1}}{h} \cdot\left(\frac{4 h_{1}}{5 e}\right)^{2} .
$$

7. Remarks about perfect domes. Let $\mathfrak{x}(u, v)=(x(u, v), y(u, v)$, $z(u, v)$ ) be a solution of (4.29) and (4.30), bounded by $\Gamma$. Obviously, the "reflected" surface

$$
\begin{gathered}
\mathfrak{r}^{*}(u, v)=\left(x^{*}(u, v), z^{*}(u, v), y^{*}(u, v)\right), \\
x^{*}(u, v)=x(u, v), \quad y^{*}(u, v)=y(u, v), \quad z^{*}(u, v)=-z(u, v),
\end{gathered}
$$

satisfies the same equations, i.e., 


$$
\begin{gathered}
L x^{*}=0, \quad L y^{*}=0, \quad L z^{*}=\frac{1}{2 z^{*}}\left|\nabla \mathfrak{x}^{*}\right|^{2}, \\
\mathfrak{x}_{u}^{* 2}=\mathfrak{r ্ v}_{v}^{* 2}, \quad \mathfrak{C}_{u}^{*} \cdot \mathfrak{r}_{v}^{*}=0, \\
L=\Delta+\Lambda, \quad \Lambda=\frac{z_{u}^{*}}{z^{*}} \partial_{u}+\frac{z_{v}^{*}}{z^{*}} \partial_{v} .
\end{gathered}
$$

Therefore, $\mathrm{r}^{*}(u, v)$ is also a stationary surface for the functional $J$ but bounded by the reflected curve $\Gamma^{*}$ (the definition of which is obvious). In fact, if $x(u, v)$ is a solution of the minimum problem

$$
J \longrightarrow \min \text { on } \mathfrak{F}(\Gamma), \quad \Gamma \subset \mathscr{K}
$$

where $\subseteq(\Gamma)$ is defined as in the beginning of $\S 3$, with $\mathscr{K}=\{z \geqq \varepsilon\}$, $\varepsilon>0$, then $\mathfrak{r}^{*}(u, v)$ solves

$$
J \longrightarrow \max \text { on } \mathfrak{J}^{*}\left(\Gamma^{*}\right), \quad \Gamma^{*} \subset \mathscr{K}^{*},
$$

where $\mathfrak{C}^{*}\left(\Gamma^{*}\right)$ is defined by $\mathfrak{C}^{*}\left(\Gamma^{*}\right)=\left\{\mathfrak{r}^{*} \in H_{2}^{1}\left(\mathscr{B}, \boldsymbol{R}^{3}\right): \mathfrak{x}^{*}(\mathscr{B}) \subset \mathscr{K}^{*}\right.$, $\mathfrak{x}^{*}: \partial . \mathscr{B} \rightarrow \Gamma^{*}$ is continuous and weakly monotonic $\}$,

$$
\mathscr{K}^{*}=\left\{z^{*} \leqq-\varepsilon\right\}, \quad \varepsilon>0 .
$$

However, whereas $z(u, v) \geqq 0$ is a subsolution of $L, z^{*}(u, v)$ is a supersolution. That is, $\mathfrak{x}(u, v)$ is hanging below $\Gamma$ (more precise: below $\left.h_{\Gamma}\right), x^{*}(u, v)$ is standing above $\Gamma^{*}$ (precisely: above $-h_{\Gamma}$ ). Suppose now that we have found a "hanging solution" $x(u, v)$ bounded by $\Gamma$. Let us build it from a homogeneous material like concrete with uniform thickness the material being incompressible to a high degree of accuracy. Turning it upside down we obtain a "standing solution" which - according to Professor Frei Otto - may serve as "perfect cupola" since it has a particular static behavior.

\section{REFERENCES}

1. W. K. Allard, On the first variation of a varifold, Annals of Math., 95 (1972), 417-491.

2. Joh. Bernoulli, Problema novum ad cuius solutionem mathematici invitantur, Acta Eruditorum Lipsiae (1696), and also: Opera omnia I, Lausannae at Genevae (1942), 161.

3. C. B. Biezeno und R. Grammel, Technische Dynamik, Bd. I, Springer.Verlag, Berlin. Göttingen-Heidelberg, 1953.

4. O. Bolza, Gauss und die Variationsrechnung, C. F. Gauss, Werke Bd. X 2, Abh., 5, Göttingen-Berlin, Springer, 1922-33.

5. O. Bolza, Vorlesungen über Variationsrechnung Leipzig, B. G. Teubner, 1909.

6. M. Chicco, Principio di massimo forte per sottosoluzioni di equazioni ellittiche di tipo variazionale, Bull. Un. Mat. Ital., (3) 22 (1967), 368-372.

7. G. Darboux, Lecons sur la théorie generale des surfaces et les applications géométriques du calcul infinitesimal, Vol. II. Paris, Gauthier-Villars 1915, Note II, 544-555.

8. D. Gilbarg and N.S. Trudinger, Elliptic partial differential equations of second order, Berlin-Heidelberg-New York, Springer, 1977. 
9. R.D. Gulliver, Regularity of minimizing surfaces of prescribed mean curvature, Annals of Math., (2) $\mathbf{9 7}$ (1973), 275-305.

10. F. Hadamard, Lecons sur le calcul des variations, Paris, Hermann et fils, 1910.

11. E. Heinz and S. Hildebrandt, Some remarks on minimal surfaces in Riemannian manifolds, Comm. Pure Appl. Math., 23 (1970), 371-377.

12. S. Hildebrandt, Maximum principles for minimal surfaces and for surfaces of continuous mean curvature, Math. Z., 128 (1972), 253-269.

13. On the regularity of solutions of two-dimensional varational problems unth obstructions, Comm. Pure Appl. Math., 25 (1972), 479-496.

14. Interior $C^{1+\alpha}$-regularity of solutions of two-dimensional variational problems with obstacles, Math. Z., 131 (1973), 233-240.

15. S. Hildebrandt and H. Kaul, Two-dimensional variational problems with obstructions, and Plateau's problem for H-surfaces in a Riemannian manifold, Comm. Pure Appl. Math., 25 (1972), 187-223.

16. F. H. Jellet, An Elementary Treatise on the Calculus of Varlations, Dublin, 1850.

17. F. H. Michael and L. M. Simon, Sobolev and mean value inequalities on generalized submanitolds of $\boldsymbol{R}^{n}$, Comm. Pure Appl. Math., 26 (1973), 361-379.

18. C. B. Morrey, The problem of Plateau on a Riemannian manifold, Annals of Math., 49 (1948), 807-851.

19. - Multiple Integrals in the Calculus of Variations, Berlin-Heidelberg-New York, Springer, 1966.

20. E. Tausch, A class of variational problems with linear growth, Math. Z., 164 (1978), 159-178.

21. F. Tomi, Variationsprobleme vom Dirichlet-Typ mit einer Ungleichung als Nebenbedingung, Math. Z., 128 (1972), 43-74.

Received March 3, 1980. The first author is indebted to SFB 40 for offering its facilities and providing financial support during his stay in Bonn, and the second autho is grateful for the hospitality of the Institute for Advanced Study in Princeton. The preparation of this manuscript was supported in part by NSF grant MCS 77-18723 (02).

Universitat Bonn

Wegelerstrasse 10

5300 Bonn, Germany 


\section{PACIFIC JOURNAL OF MATHEMATICS}

\section{EDITORS}

DONALD BABBITT (Managing Editor)

University of California

Los Angeles, CA 90024

Hugo Rossi

University of Utah

Salt Lake City, UT 84112

C. C. MOORE and ANDREW OGG

University of California

Berkeley, CA 94720
J. DUGUNDJI

Department of Mathematics

University of Southern California

Los Angeles, CA 90007

R. FinN and J. Milgram

Stanford University

Stanford, CA 94305

\section{ASSOCIATE EDITORS}
E. F. BECKENBACH
B. H. NeumanN
F. WOLF
K. YoSHIDA

\section{SUPPORTING INSTITUTIONS}

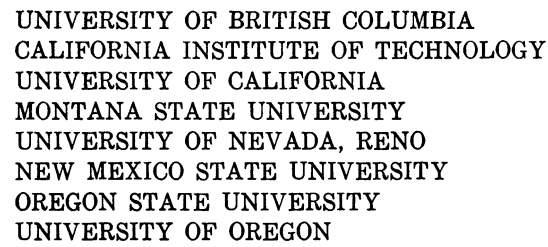

UNIVERSITY OF BRITISH COLUMBIA CALIFORNIA INSTITUTE OF TECHNOLOGY UNIVERSITY OF CALIFORNIA MONTANA STATE UNIVERSITY UNIVERSITY OF NEVADA, RENO NEW MEXICO STATE UNIVERSITY OREGON STATE UNIVERSITY UNIVERSITY OF OREGON

\author{
UNIVERSITY OF SOUTHERN CALIFORNIA \\ STANFORD UNIVERSITY \\ UNIVERSITY OF HAWAII \\ UNIVERSITY OF TOKYO \\ UNIVERSITY OF UTAH \\ WASHINGTON STATE UNIVERSITY \\ UNIVERSITY OF WASHINGTON
}

The Supporting Institutions listed above contribute to the cost of publication of this Journal, but they are not owners or publishers and have no responsibility for its content or policies.

Mathematical papers intended for publication in the Pacific Journal of Mathematics should be in typed form or offset-reproduced, (not dittoed), double spaced with large margins. Please do not use built up fractions in the text of the manuscript. However, you may use them in the displayed equations. Underline Greek letters in red, German in green, and script in blue. The first paragraph or two must be capable of being used separately as a synopsis of the entire paper. Please propose a heading for the odd numbered pages of less than 35 characters. Manuscripts, in triplicate, may be sent to any one of the editors. Please classify according to the scheme of Math. Reviews, Index to Vol. 39. Supply name and address of author to whom proofs should be sent. All other communications should be addressed to the managing editor, or Elaine Barth, University of California, Los Angeles, California, 90024.

50 reprints to each author are provided free for each article, only if page charges have been substantially paid. Additional copies may be obtained at cost in multiples of 50 .

The Pacific Journal of Mathematics is issued monthly as of January 1966. Regular subscription rate: $\$ 84.00$ a year (6 Vols., 12 issues). Special rato: $\$ 42.00$ a year to individual members of supporting institutions.

Subscriptions, orders for numbers issued in the last three calendar years, and changes of address shoud be sent to Pacific Journal of Mathematics, P.O. Box 969, Carmel Valley, CA 93924, U.S.A Old back numbers obtainable from Kraus Periodicals Co., Route 100, Millwood, NY 10546.

PUBLISHED BY PACIFIC JOURNAL OF MATHEMATICS, A NON-PROFIT CORPORATION

Printed at Kokusai Bunken Insatsusha (International Academic Printing Co., Ltd.). 8-8, 3-chome, Takadanobaba, Shinjuku-ku, Tokyo 160, Japan. 


\section{Pacific Journal of Mathematics \\ Vol. 88, No. $2 \quad$ April, 1980}

Reinhold Böhme, Stefan Hildebrandt and Engelbert Tausch, The two-dimensional analogue of the catenary ................. 247

Jean Ellen Taylor, Nonexistence of F-minimizing embedded disks ........ 279

Claus Gerhardt, A free boundary value problem for capillary surfaces ..... 285

Enrico Giusti, Generalized solutions for the mean curvature equation . . . . . 297

Jin-Tzu Chen, On the existence of capillary free surfaces in the absence of gravity.................................... 323

Leon M. Simon, Regularity of capillary surfaces over domains with corners.......................................... 363

Nicholas Jacob Korevaar, On the behavior of a capillary surface at a re-entrant corner.................................... 379

Henry Wente, The symmetry of sessile and pendent drops ............ 387

E. Gonzalez, Umberto Massari and I. Tamanini, Existence and regularity for the problem of a pendent liquid drop ...................... 399

Henry Wente, The stability of the axially symmetric pendent drop ........ 421

David Siegel, Height estimates for capillary surfaces . . . . . . . . . . . . 471

Bruce Edward Turkington, Height estimates for exterior problems of capillarity type ................................ 517

Robert Finn, The sessile liquid drop. I. Symmetric case .............. 541 